\title{
1 Locally distributed abstraction of temporal distance in human parietal
}

2 cortex

3

4 Qun $\mathrm{Ye}^{1}$, Yi Hu${ }^{1}$, Yixuan $\mathrm{Ku}^{1,3}$, Kofi Appiah", Sze Chai Kwok * 1,2,3

5

6 Short title: Precuneus and temporal context memory

7

8

9

10

11

12

13

14

15

16

1. Shanghai Key Laboratory of Brain Functional Genomics, Key Laboratory of Brain Functional Genomics Ministry of Education, School of Psychology and Cognitive Science, East China Normal University, Shanghai 200062, China

2. Shanghai Key Laboratory of Magnetic Resonance, East China Normal University, Shanghai 200062, China

3. NYU-ECNU Institute of Brain and Cognitive Science at NYU Shanghai, Shanghai 200062, China

4. Department of Computing, Faculty of ACES, Sheffield Hallam University, Sheffield S1 2NU, England

*Corresponding author: sze-chai.kwok@st-hughs.oxon.org (Sze Chai Kwok) 


\section{Abstract}

An enduring puzzle in the neuroscience of memory is how the brain parsimoniously situates past events by their order in relation to time. By combining functional MRI, and representational similarity analysis, we reveal a multivoxel representation of time intervals separating pairs of episodic event-moments in the posterior medial memory system, especially when the events were experienced within a similar temporal context.

We further show such multivoxel representations to be vulnerable to disruption through targeted repetitive transcranial magnetic stimulation and that perturbation to the mnemonic abstraction alters the neural—behavior relationship across the wider parietal memory network. Our findings establish a mnemonic "pattern-based” code of temporal distances in the human brain, a fundamental neural mechanism for supporting the temporal structure of past events, assigning the precuneus as a locus of flexibly effecting the manipulation of physical time during episodic memory retrieval. 


\section{Introduction}

Precuneus and temporal context memory

5

Time in physics is operationally defined as "what a clock reads". While the passage of time between two moments can be precisely measured by a quartz crystal oscillator or biologically registered by distributed sets of brain regions across intervals of time $(1,2)$, how the human brain can parsimoniously situate past events by their order in relation to time and abstract temporal distances separating events in long-term, episodic memory is incompletely understood (3).

Representations of brief elapsed time can be inferred from single neuron activities in the primate brain (4-6). Time-registering neurons are found to code time with high precision in the cortico-basal ganglia circuits (5), inferior parietal cortex (4) and medial temporal lobe (6) across short timescales. Recent work in rats has provided evidence that temporal information is encoded across time scales from seconds to hours within the overall population state of the lateral entorhinal cortex (7). In contrast, when complex, coherent experiences become consolidated into long-term memories (8), the neural circuits that build time representations as an infrastructure for episodic retrieval are theorized to be distinct from those implicated in hippocampal-dependent encoding $(9,10)$ and retrieval $(11,12)$, and from those during transient temporal processing $(4-$ 6). For the recollection of long-term autobiographical memories or episodic events, the posterior medial (PM) memory system, including hippocampus, precuneus and angular gyrus, plays an instrumental role (13). Event representations in these regions generalized across modalities (e.g., EEG and MRI) and domains (e.g., perception and memory) (14-16). 
Temporal representation is intertwined with the construct of context. A prominent memory model posits that item representations are linked to a changing "context" at encoding, such that a common retrieved context is triggered during recall for items that were experienced within a similar temporal context (17). However, the critical issue of how elapsed time between pairs of long-term episodic events - and its interplay with the encoding context - is represented by the PM system has yet to be addressed. Here we investigated the abstraction, at a macro-anatomical level, of temporal distances that were encoded more than 24 hours previously $(18,19)$, and determined how several members of this large cortical system are differentially implicated in this putative mnemonic function (20).

Combining functional magnetic resonance imaging (fMRI) with an interactivevideo memory paradigm and a temporal order judgement task (TOJ; Fig 1A) - a validated paradigm to study neural correlates underpinning temporal distances between units of memory traces $(10,18,19)$-we adopted a two-forked protocol to ascertain how temporal distances separating pairs of past moments-in-time are represented in the human neocortex. On the one hand, we identified a locally distributed neural representation characterizing the neural patterns of retrieving temporal distances using a multivariate searchlight representational similarity analysis (RSA) (21). We parametrized a large set of pairs of event-moments geometrically separated by varying temporal intervals and applied RSA to compare neural representational dissimilarity matrices (RDM) with a number of parametric, condition-rich hypothetical/candidate models. Applied across the entire brain, the searchlight approach identifies local multi- 
voxel patterns driven by structured co-activation at a voxel level within the size of the 9-mm radius spherical searchlight, thereby giving us a snapshot of the locally distributed neural architecture supporting temporal order judgements. On the other hand, to enhance the causal strength of the anatomical associations thereby revealed, we focally disrupted the identified critical region with repetitive transcranial magnetic stimulation (rTMS, Fig 1F), seeking to confirm its functional necessity for mediating the distributed representation of temporal distances. The spatial scale of rTMS-induced disruption is comparable to that of our chosen searchlight, rendering it an optimal tool for targeted, reversible disruption of the distributed representation of interest.

For memory encoding, participants played an interactive video game containing seven distinct yet related chapters, each in the range of tens of minutes on day 1 (S1

Fig, S1 Table). By the nature of the video game, within chapter segments contained more coherent narrative strands than those across chapters, yet all chapters were connected by a common plot. After a 24-hour retention period (day 2), on each trial, participants judged the temporal order of two images (extracted from their individuallyplayed video game, Fig 1B), depicting two time-points in their encoded memory, while their blood-oxygen-level-dependent (BOLD) activity was measured (TOJ task, Fig 1C). Assuming a scale-free temporal memory representation (22), we manipulated the between-images temporal distances (TD) for all pairs of images so that the TD distribution adhered to a power function permitting scale-invariance across subjects (23) (60 levels of TD, Fig 1D). To test the interaction effect between TD and its encoding context, we manipulated the factor “context” by controlling whether the paired images 
bioRxiv preprint doi: https://doi.org/10.1101/249904; this version posted October 5, 2018. The copyright holder for this preprint (which was

not certified by peer review) is the author/funder, who has granted bioRxiv a license to display the preprint in perpetuity. It is made available under aCC-BY 4.0 International license.

Precuneus and temporal context memory

100 presented at TOJ task were extracted from the same chapters or two adjacent chapters

101 of the video game while keeping the 60 TDs fully matched between the two conditions

102 (Within-chapter vs. Across-chapter, Fig 1E).

103

A

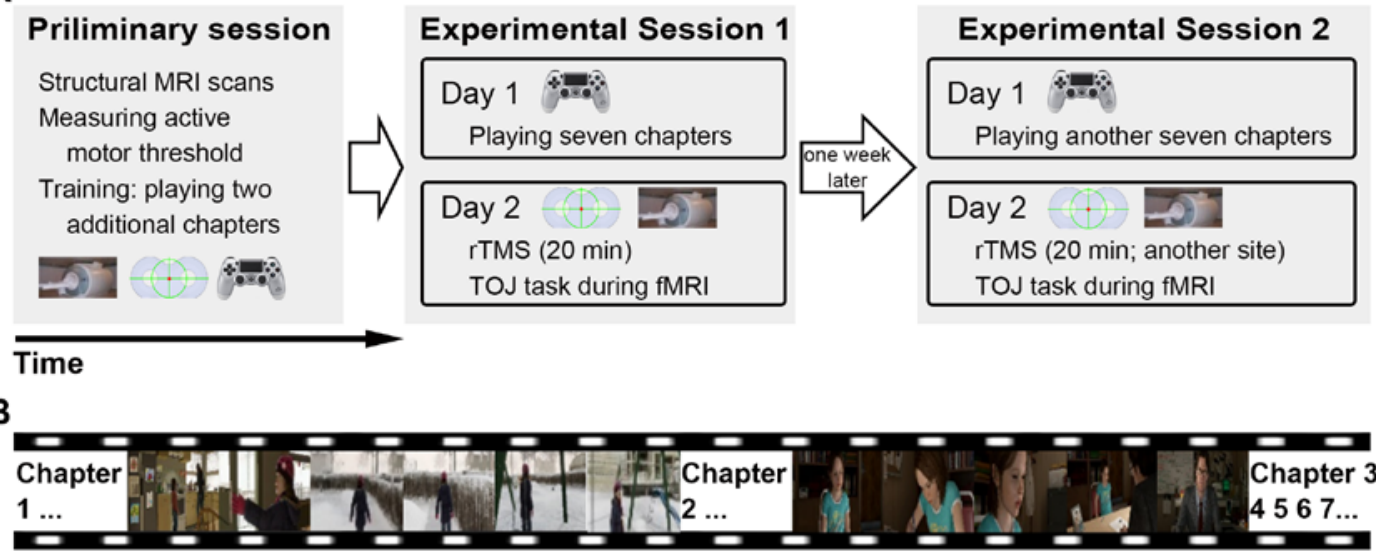

C

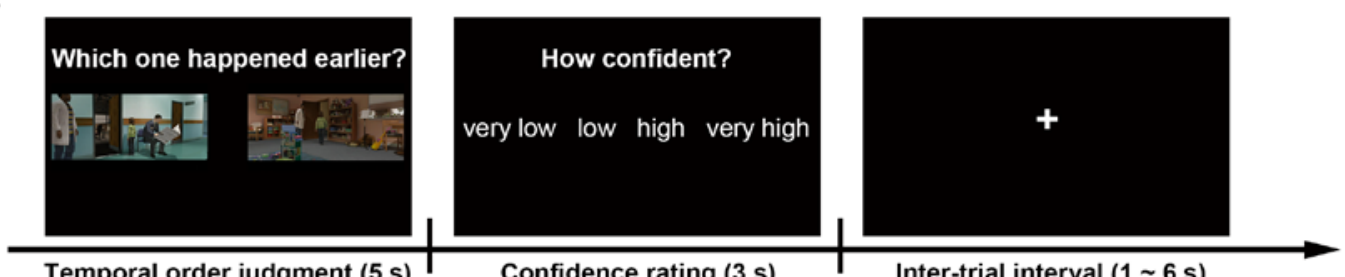

D

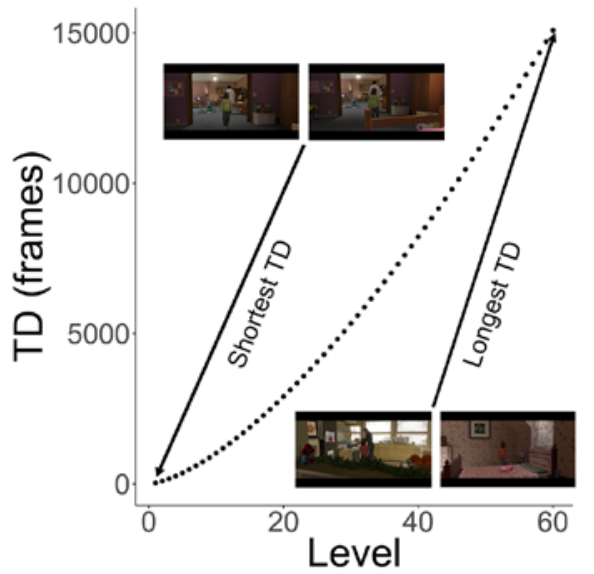

$\mathrm{E}$

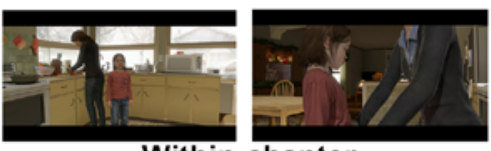

Within-chapter

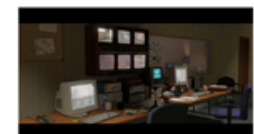

$\mathbf{F}$

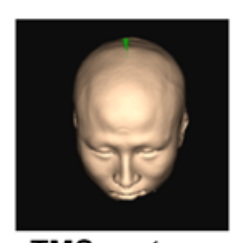

TMS-vertex

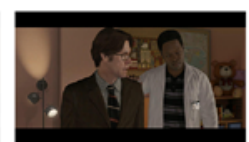

chapter

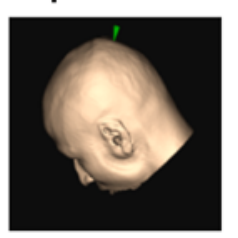

TMS-precuneus

105 Fig 1. Experiment overview. (A) In experimental sessions 1 and 2, participants played a video 
one of two cortical sites before performing a temporal order judgement task during fMRI. Order of TMS sites (within-subjects) and choices of video game chapters were counterbalanced across subjects (S1 Table). The two experimental sessions were conducted on different days to minimize rTMS carry-over effects (mean separation $=8$ days). Participants underwent structural MRI scans and familiarized themselves with the gameplay using a console prior to experimental sessions proper. (B) Gameplay video: each encoding session consisted of seven chapters (S1 Fig). (C) Temporal order judgement task. Participants chose the image that happened earlier in the video game and reported their confidence level. (D) 60 levels of temporal distances (TD) were generated for each subject according to their subject-specific video-playing duration. Although the absolute TD were different across subjects (S1 Fig), we ensured it to be scale-invariant using a power function during image selection. Actual TDs from one subject (subj01) are shown. (E) Two pairs of images were extracted from the same chapter (Within-chapter) or two adjacent chapters (Across-chapter). The 60 levels of TD were fully matched within-subjects for these two conditions. Note that scenes depicted in Within-chapter tended to be more contextually similar than those depicted in Across-chapter. (F) TMS stimulation sites, superimposed onto one subject’s MRI-reconstructed skull, are marked by a green pointer. The MNI coordinates for precuneus stimulation: $\mathrm{x}, \mathrm{y}, \mathrm{z}=6,-70,44$.

\section{Results}

\section{Behavioral results}

We first looked into the interaction effect between TD and encoding context and 
$\left(F_{(1,16)}=25.53, P<0.001, \eta^{2}=10.02 \%\right)$ and a significant two-way interaction effect

between TD and Context $\left(F_{(1,16)}=5.97, P=0.026, \eta^{2}=2.71 \%\right)$. Such interaction effect

was driven by a significant difference in accuracy between short and long TD in Within-

$$
\text { 1.61, } P=0.117) \text { (Fig 2A). }
$$

A similar two-way interaction effect was found in reaction times $\left(F_{(1,16)}=24.21\right.$, $P<0.001, \eta^{2}=1.16 \%$ ), with longer RT in short than in long TD in Within-chapter condition $\left(t_{(33)}=-3.33, P=0.002\right)$ but longer RT in long TD condition in Across-chapter condition $\left(t_{(33)}=2.83, P=0.008\right)($ Fig $2 B)$. No three-way interaction effects were found

144 in either of the measures ( $P$ s $>0.05$ ). A similar behavioral pattern was found in the 145 measure of confidence level (S2 Fig). In terms of TMS effect on behavioral measures, 146 TMS to the precuneus resulted in slowed reaction times (main effect of TMS: $F_{(1,16)}=$ $1475.34, P=0.035, \eta^{2}=2.65 \%$ ) as compared with the TMS-vertex condition. 
Precuneus and temporal context memory

A

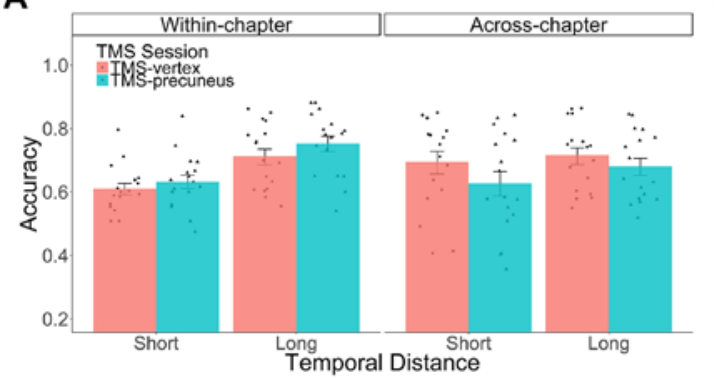

B

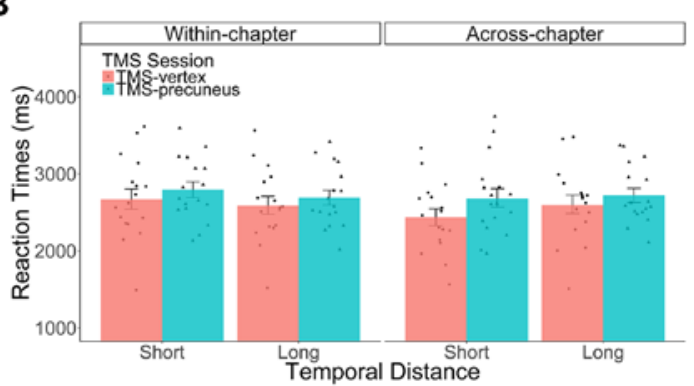

Fig 2. Behavioral results. (A) Accuracy: The difference between short and long temporal distances was more pronounced for Within-chapter condition than for Across-chapter condition irrespective of TMS stimulation. (B) Reaction times: The differences between short and long temporal distances were in an opposite way between the Within-chapter condition and the Across-chapter condition. TMS stimulation resulted in longer reaction times in TOJ task. Dots denote behavioral performance per subject. Error bars denote the SEM over subjects.

\section{Functional MRI results}

Representation of temporal distances. First and foremost, we searched for neural representations which might resemble the matrix of temporal distances using searchlight representational similarity analysis (21). Without a priori bias for any region of interest, we searched the entirety of the cortex using an RDM consisting of 60 levels of logarithmically-transformed subject-specific TD (Fig 3A) and identified clusters of voxels that contain information of the set of geometrically defined temporal distances in memory (see Materials and methods and S3 Fig). Within this $60 \times 60$ RDM of temporal-distances, we revealed that the neural pattern of judging the temporal order of a pair of memories separated with a given temporal distance is more similar to other temporal order judgements which also enclosed temporal distances of a 
comparable scale. These voxels were in the posteromedial parietal areas, bilateral angular gyri, and middle frontal gyri (Fig 3B, S2 Table).

The temporal-distance memory representation could be confounded by perceptual

171 similarity in each pair of images. To address this concern, we conducted six separate

RSAs, in which we indexed perceptual similarity between the image-pairs by six different metrics, some drawn from the visual categorization models referred by Greene et al (24) and Aminnoff et al (25). The six RDMs are Red Green and Blue (RGB) crosscorrelation RDM, RGB-intensity RDM, RGB-histogram RDM, Scale Invariant Feature similar representation was observed in the posteromedial parietal cortex using these candidate RDMs except the SURF RDM (S4B-G Fig). Importantly, to further exclude the contribution of these additional perceptual properties, we conducted a whole brain searchlight correlation between the TD RDM and the brain response pattern, this time regressing out the six perceptual RDMs using a partial Spearman correlation analysis method. This partial correlation analysis showed that the effects of abstraction of TD distributed in the posteromedial parietal areas remained even after removing the influences of all of these perceptual properties, suggesting that TD multivoxel representation could not be driven simply by the perceptual properties (S4J Fig). 
Despite the space-time correlation in the encoding material (S8 Fig), subject-specific

Situational Changes RDMs explained very little variances in comparison to our temporal-distance RDM (S4A Fig). We also confirmed that participants' behavioral performance RDMs, namely reaction times and accuracy, could not explain the putative temporal-distance representation (S4H-I Fig).

Having identified a multivariate pattern underlying the temporal memory abstraction in the posterior medial parietal cortex, we asked further whether there were voxels whose activities change monotonically as a function of temporal distance using a standard univariate approach, irrespective of its multivoxel characteristics. A wholebrain parametric modulation analysis revealed TD-specific BOLD signals in a cluster within the posteromedial region, including the precuneus (Fig 3C, S3 Table). This parametric relationship could not be attributed to difficulty (results were the same after trial-by-trial reaction times were regressed out, S6A Fig). Importantly, since the two types of analyses extracted two different kinds of neural information, their overlap in the precuneus jointly confirms the critical involvement of this region. We accordingly created a conjunction map (Fig 3D), so that both the multivariate and univariate results underlying the temporal distance abstraction would be available for the next analysis. 
214 variance far better than the other candidate RDMs, such as SC RDM, six perceptual

215 RDMs and two behavioral RDMs (Fig 3E-F). These results showed that the neural

A

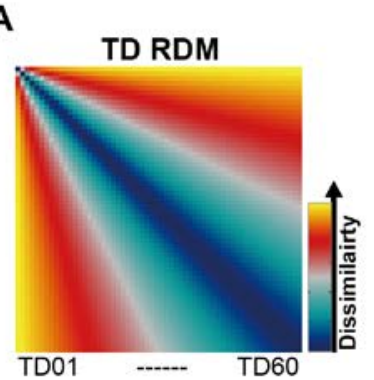

B

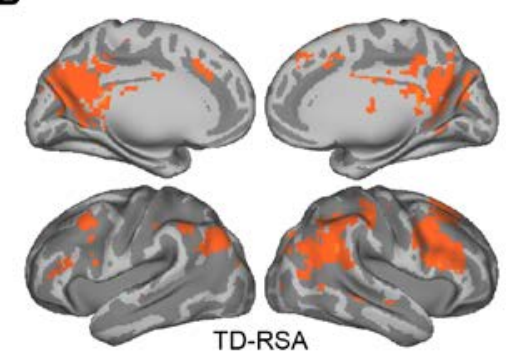

C

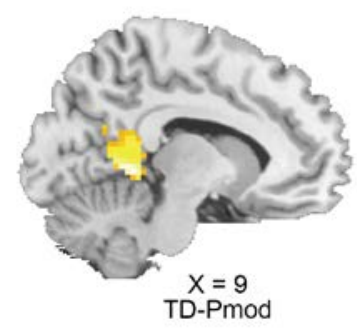

D

E

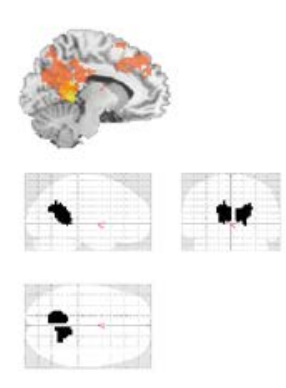

RSA $\cap$ Pmod

F
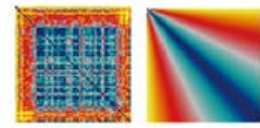<smiles>C1CCCC1</smiles>
s

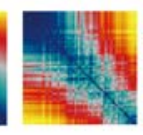

c

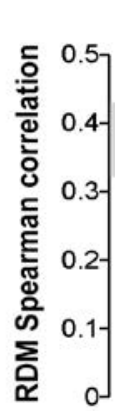

$\left.\begin{array}{l}0.5 \\ 0.4 \\ 0.3 \\ 0.2 \\ 0.1 \\ 0\end{array}\right]$

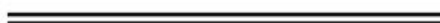

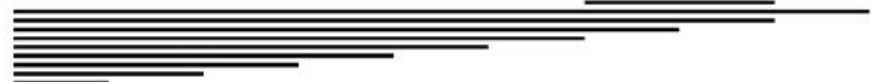$$
\text { nemonic representation of temporal distances. }
$$

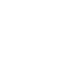


(Pmod). The intensity of these voxels, primarily in the left precuneus, increased as a function

(S2\&3 Table). (E) Inferential comparisons of multiple model representations. Several

RDM extracted from the conjunction mask. TD RDM was the best model among these candidate RDMs to explain the variances in the neural reference RDM. Horizontal lines over an unknown true model, given the noise in the data. Asterisks indicate a significant Spearman correlation between the subject-specific reference RDM and candidate RDMs. Error bars indicate the SEM over participants. * $P<0.05$, FDR-corrected. (F) Reference (neural) RDM

239 and candidate RDMs. The reference RDM was estimated using the BOLD responses elicited

240 by the stimuli during fMRI in accordance to the 60 TD levels in the conjunction region. We averaged 17 subject-specific neural reference RDMs for display purpose. SC RDM was

243 Fig). Perceptual RDMs (RGB cross-correlation, RGB intensity, RGB histogram, SIFT, SURF, 
HOG) were computed based on image properties (see details in Materials and methods).

Behavioral RDMs (RT and ACC) were computed based on the behavioral performance of reaction times and percentage correct for each trial (see details in Materials and methods).

MRI results are displayed at $P_{\text {uncorrected }}<0.001$.

TD representation is context-dependent. To test the hypothesis that the TD-

neural pattern similarity index to be higher when the two images are extracted from a

"similar context” than when they are from two "different contexts", we ran a new

searchlight RSA, now separately for the Within-chapter and Across-chapter trials. The

but not in the Across-chapter condition (Fig 4A, right). The voxels identified by the

searchlight RSA were in the precuneus, retrosplenial cortex, and angular gyri bilaterally.

For statistical inference, we extracted the similarity index within the

aforementioned conjunction mask (RSA and pmod maps), using a bias-free leave-one-

subject-out method (see Materials and methods). In line with our prediction, the 
(Fig 4B, left). By contrast, the TD RDM (and all other candidate RDMs) failed to explain the neural reference RDM in the Across-chapter condition (Fig 4B, right).

269 analysis. The beta-estimates $(\beta)$ from a pmod analysis using TD as a regressor were significantly higher in the Within-chapter condition compared to the Across-chapter condition (Fig 4C). These results were consistent in a control analysis while RT were

272 regressed out from the pmod analysis (S6B Fig). This confirmed that the mnemonic

273 representation of temporal distances was determined by whether the pairs of images

274 were experienced within a similar context, corroborating the interaction between temporally- and semantically-defined factors observed during memory encoding (9) 276 and retrieval (12). 
A
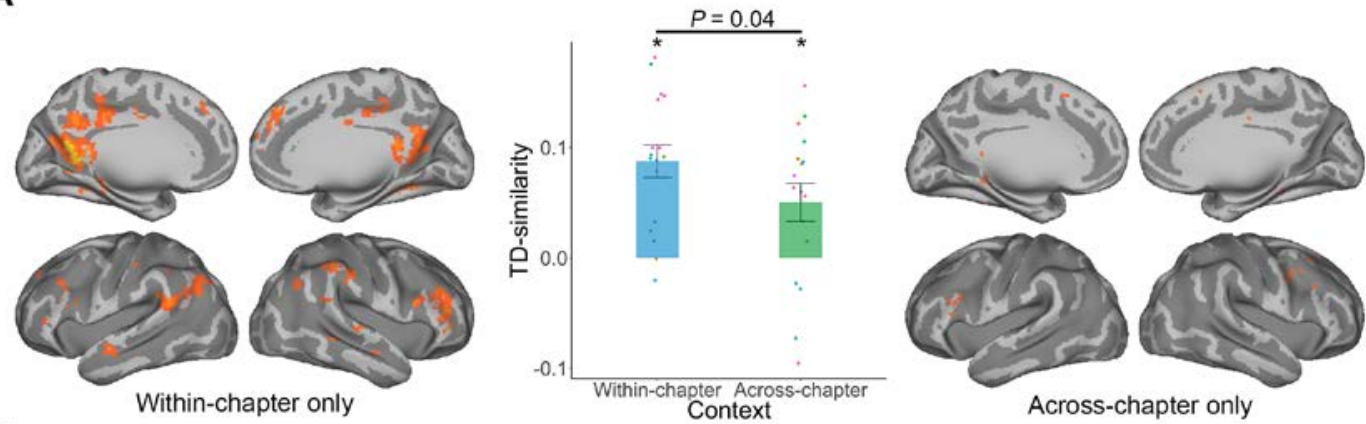

B
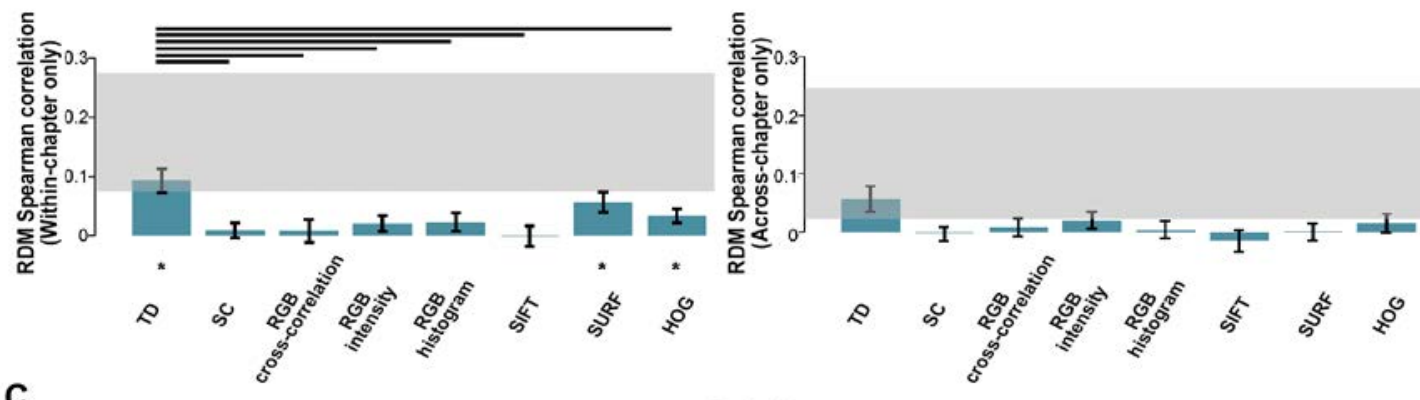

C
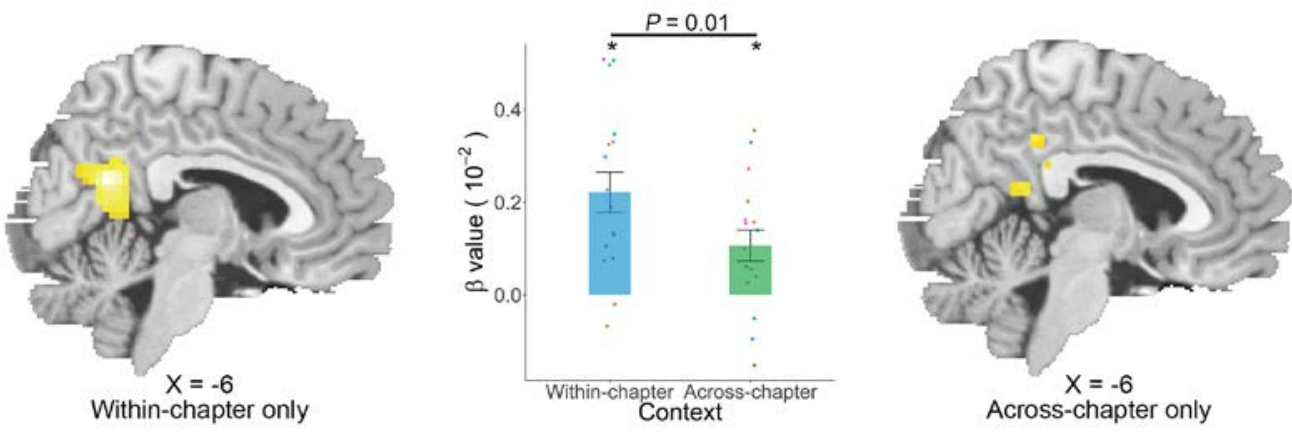

Fig 4. TD representation is context-dependent. (A) Stronger multivoxel similarity

representing TD variation in the Within-chapter condition than in the Across-chapter condition

in the conjunction region (middle panel, one-tailed: $P=0.04$ ). Each same color dot represents one subject. Error bars denote the SEM over subjects. ${ }^{*} P<0.05$, FDR-corrected, one-sample t-test against zero. (B) Separate model comparisons showed the TD RDM is the best model to explain neural variances in the Within-chapter condition (although SURF RDM and HOG RDM are also related to the neural reference RDM), whereas all the candidate RDMs could not explain the neural variances in the Across-chapter condition. (C) Stronger parametric activation intensity in the Within-chapter condition than in the Across-chapter condition. 
pivotal role of the precuneus in this memory operation we deployed a disruptive technique, strategically targeting the precuneus with repetitive transcranial magnetic stimulation to interrogate changes on both neural and behavioral levels (within-subjects: representation of TD disappeared following TMS on the precuneus, either considering the Within-chapter condition (Fig 5A) or collapsing Within-chapter and Across-chapter conditions. Model comparison results showed that the correlation values between all the candidate RDMs and neural reference RDM now failed to reach statistical significance even when only considering Within-chapter trials alone (Fig 5B). In contrast, the activation-based Pmod analyses showed that TMS to the precuneus did not induce any discernable changes in the univariate BOLD intensity (S6C Fig) possibility that there might be differences in the patterns of neural activity associated with the abstraction of temporal distances in the sub-regions of the PM memory network (13). Based on our main MRI results (Fig 3B) and previous work on the parcellation of the PM memory network (20), we have chosen six anatomical regionsof-interest in the PM memory network (ROIs: bilateral precuneus, bilateral angular gyrus and bilateral hippocampus; see Materials and methods), together with the primary visual region (entire occipital cortex) as a control, to more finely characterize the disruptive effect caused by the TMS. 
311 pattern similarity in the left precuneus was significantly weakened following TMS to

312 the precuneus specifically for Within-chapter condition (Fig $\mathbf{5 C}$, one tailed: $P=0.02$;

313 to a lesser extent, also the right precuneus and left angular gyrus, S7A\&D Fig).

314 Specifically, we found that changes in individuals' neural-TD pattern similarity in the

315 vertex condition to be associated positively with their TOJ memory performance in this

316 key region (Fig 5C, $r=0.60, P=0.04$; also in the left hippocampi, see S7B Fig),

317 implying these multivoxel representations are relevant neurobiological prerequisites for

318 the ability to support temporal order judgement. In the experimental condition wherein

319 we disrupted the precuneal activity with magnetic field prior to retrieval, changes in

320 neural-behavioral correlation were resulted (Fig 5C, right panel; comparison between

321 two correlations: $z=2.07, P=0.04)$. Since the focal perturbation altered the mnemonic

322 representation across parts of the PM system implicating the precuneus, the angular

323 gyri and the hippocampi, the putative disruption might have been effective through

324 inducing alternation in functional connectivity between multiple regions, or more

325 globally throughout the entire parietal memory network (31, 32). 
A

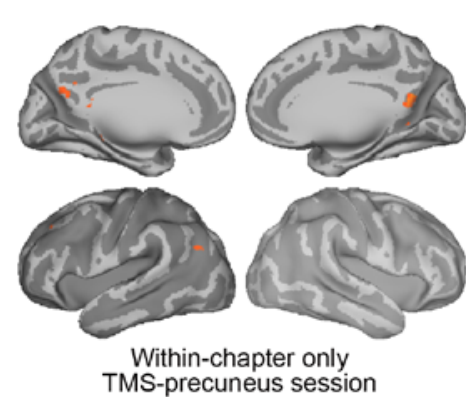

C
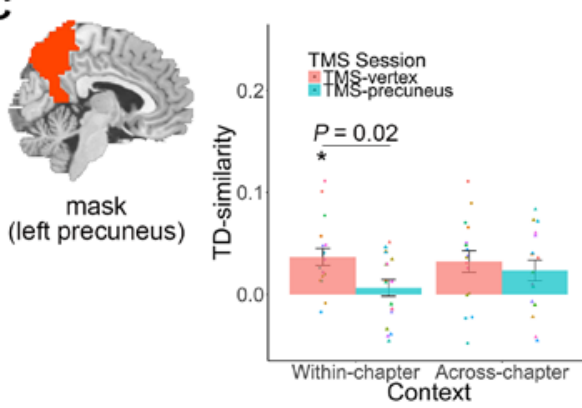

\section{B}
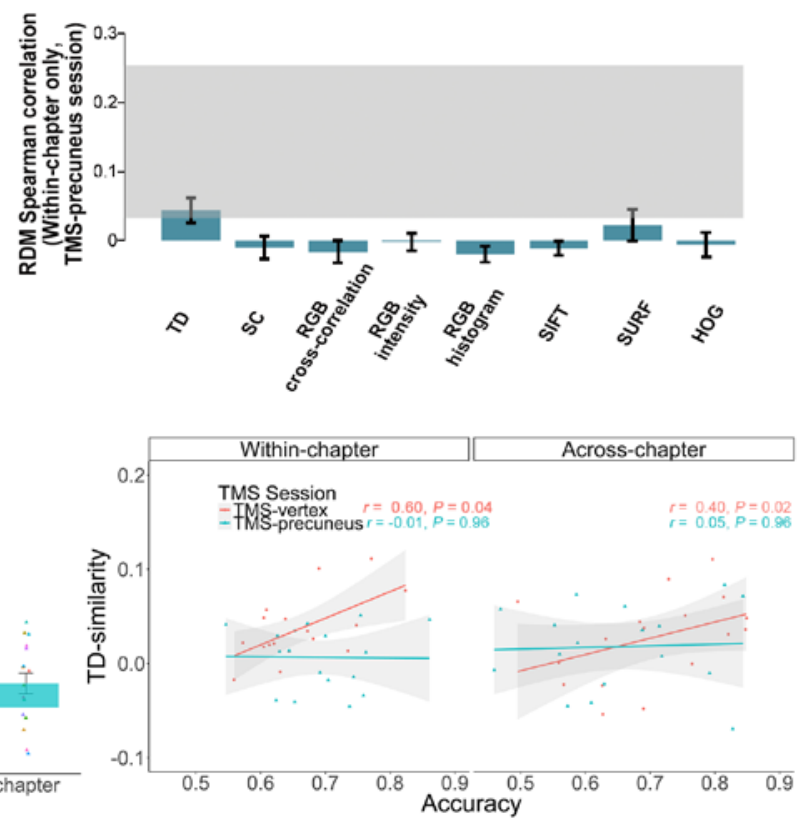

Fig 5. TMS reduced the precuneal representation of TD. TMS reduced the precuneal

representation of TD. (A) Very few voxels survived in the searchlight RSA after TMS on the precuneus (voxel level: $P_{\text {uncorrected }}<0.001$; cluster level: $P_{\text {FWE }}<0.05$ ). (B) Model comparisons showed that the candidate RDMs explain little neural variances after TMS on the precuneus. (C) We used left precuneus as a mask to extract TD-similarity values from subjects and found that TMS-precuneus, compared to the TMS-vertex, reduced the TD representation specifically in the Within-chapter condition (middle panel, one tailed: $P=0.02$ ). Further correlation analyses showed that TMS on the precuneus weakened the significant neural-behavioral correlation (TMS-vertex session: $r=0.60, P=0.04$; TMS-precuneus session: $r=-0.01, P=$ 0.96; comparison between two correlations: $z=2.07, P=0.04) . P$ values are FDR-corrected.

\section{Discussion}

By combining fMRI, rTMS and multivoxel pattern analysis with a novel interactive 
temporal distance between pairs of episodic events in the human parietal cortex and showed that BOLD intensity of posteromedial cortex, especially the precuneus, varied as a function of temporal distance. We showed that such information carried by these regions is more pronounced within a similar context and such representations reduced significantly after the precuneus was perturbed. We further revealed that the precuneal representation of TD is associated with subjects' memory performance, especially when two images for temporal order judgment were extracted from the same context. images were associated within a similar context. The parietal representation of temporal distances between pairs of episodic events observed here, as revealed by both univariate and multivariate pattern analyses, might act in parallel with hippocampal cells that code specific moments in time or temporal positions (33), or act independently as a separate mnemonic establishment of episodes over and above the hippocampal memory ensemble (34).

Previous studies have associated neural similarity in the hippocampus with both spatial and temporal aspects of episodic memory (29, 35, 36). Studies on rats have also 359 showed that gradually changing representation, as manipulated by temporal 360 lag/distance, is manifested in the hippocampus (10). These studies have mostly focused on neural similarities in the hippocampus (29, 36, 37), whereas here we revealed a TD representation primarily in the PM system. This discrepancy might be due to the fact that instead of using a passive paradigm (viewing movies or listening to narratives), we 
used an active paradigm (first-person perspective gameplay for encoding), which

should have implicated a distinct system upon retrieval (38)'(39) when the memories in question are akin to real-life autobiographical/episodic experiences. The consideration that a temporal order judgment task was used in which participants had to extract temporal distance for making a decision (40), and then the neural signals were assessed in relation to the actual temporal distances rather than to subjective estimation of temporal separation might have also engaged the PM memory system more heavily.

It is theoretically interesting that the multivariate representations were more vulnerable to the magnetic stimulation than the voxel-wise signal intensity (note that TMS to the precuneus did not induce any discernable changes in univariate BOLD level, see S6C Fig). These findings support our argument that memory traces that are represented during temporal order judgement are indeed conveyed in some localized multivoxel readouts housed in the PM system cortices, above and beyond the modulated changes in canonical BOLD activation. Building on extant connectivity findings between the hippocampus and neocortical regions $(13,41,42)$ and the hippocampal role in temporal context memory $(9,12)$, our demonstration of distributed pattern of temporal information in the posteromedial parietal region implied the existence of a higher level parietal mnemonic readout of temporal distances between episodic experiences.

Model comparisons incorporating several control analyses robustly confirm that such precuneal representation of temporal distances do not merely reflect some trivial effects related to task difficulty $(18,43)$ or inferred distance based on image properties. 
Although some perceptual models (such as SUFT model) might produce a similar RSA map, when we partially removed the respective influences of perceptual properties, the

effects of TD representation in the posteromedial parietal cortex were largely unaffected (S4J Fig). Moreover, in terms of the stimuli features, since our images were extracted randomly across chapters of the video game (S1C Fig), and since the TD distributions were fully matched between the Within-chapter and Across-chapter conditions, predictions made by the positional coding theory (44) would also not be sufficient to account for the differential TD representational results across these two conditions.

In summary, our multivariate searchlight results reveal that the temporal distance representations in the posterior parietal cortex, especially in the precuneus, during TOJ retrieval are determined by how temporally distant (and how similar the encoding contexts) two given event-moments the subjects had encountered $(18,19)$. We also establish that this multivoxel mnemonic abstraction is localized in the precuneal area

400 and perturbation to it alters the neural—behavior relationship across the global parietal

401 memory network, assigning this structure as a locus of flexibly effecting the 


\section{Materials and methods}

Participants. Twenty individuals participated in the study (7 female, $22.55 \pm 1.54$ years, mean \pm sd). Data from 3 subjects were excluded due to either poor performance (1 subject performed at chance level) or scanner malfunction (projector crashed during scanning for 2 subjects at TMS-vertex session), resulting in a final group of 17 subjects (7 female, $20.65 \pm 1.54$ years, mean $\pm \mathrm{sd}$ ). All subjects were unfamiliar with the video game, had normal or correct-to-normal vision and did not report neurological or psychiatric disorders or current use of psychoactive drugs. All subjects were eligible for MRI and TMS procedures based on standard MRI safety screening as well as on their answers to a TMS safety-screening questionnaire (45). No subjects withdrew due to complication from the TMS or MRI procedures, and no negative treatment responses were observed. All subjects gave written informed consent and were compensated for their participation. All procedures were performed in accordance with the 1964 Helsinki declaration and its later amendments and approved by University Committee on Human Research Protection of East China Normal University (UCHRP-ECNU). The number of participants was determined based on previous studies with similar design (9, 32).

Experimental design, stimuli, and tasks. Encoding: Interactive video game. The action-adventure video game (Beyond: Two Souls) was created by the French game developer Quantic Dream and played in the PlayStation 4 video game console 
person perspective. To ensure that the participants mastered the operational capability, they were trained to play the game with two additional game chapters (Training chapters: Welcome to the CIA, and The Embassy). The training session varied in duration depending on the dexterity of each participant on using the console (40 - 60 min per chapter). After the training session, participants played 14 chapters in total across two sessions: 7 in Experimental Session 1 and then another 7 in Session 2 (Fig 1). The video game they played were recorded and stored as a single video file in MP4 format (Chapters 1 7: My Imaginary Friend, First Interview, First Night, Alone, The Experiment, Night Session, Hauntings; Chapters 8 14: The Party, Like Other Girls, Separation, Old Friends, Norah, Agreement, Briefing; see S1 Fig).

Retrieval (scanned): Temporal Order Judgment (TOJ) task. The TOJ retrieval task required participants to choose the image that happened earlier in the video game they had encoded. The task was administrated inside an MRI scanner, where visual stimuli were presented using E-prime software (Psychology Software Tools, Inc., Pittsburgh, PA), as back-projected via a mirror system to the participant. Each trial was presented for $5 \mathrm{~s}$ during which participants performed the temporal order judgment. They were then allowed $3 \mathrm{~s}$ to report their confidence level following the memory judgement.

443 Participants performed the TOJ task using their index and middle fingers of one of their 444 hands via an MRI compatible five-button response keyboard (Sinorad, Shenzhen, China). Participants reported their confidence level (“Very Low”, “Low”, "High”, or 
counterbalanced across participants. Participants were told they should report their confidence level in a relative way and make use of the whole confidence scale.

450 Following these judgments, a fixation cross with a variable duration (1 - $6 \mathrm{~s})$ was

451 presented. Each participant completed 240 trials in each of the two experimental sessions. Participants were given 15 practice trials using paired images extracted from the two additional chapters they had played in the training session out of the scanner to 454 ensure they understand the task procedure. Participants completed a surprise recognition test after TOJ task outside scanner; data of which are not reported here. videos which the participants had played the day before. Each second in the video consisted of 29.97 static images (frames). For each game-playing session, 240 pairs of images were extracted from the seven chapters and were paired up for the task based on the following criteria: (1) the two images had to be extracted from either the same chapters or adjacent chapters (Within-chapter vs. Across-chapter); (2) the temporal 462 distance (TD) between the two images were matched between Within- and Acrosschapter condition; (3) in order to maximize the TD, we first selected the second longest 464 chapter of the video and determined the longest TD according to a power function 465 (power $=1.5$ ), at the same time ensuring the shortest TD to be longer than 30 frames. 466 We generated 60 progressive levels of TD among these pairs (each level repeated twice). 467 In sum, three within-subjects factors regarding the TOJ retrieval task were manipulated: 468 (1) 60 TD levels permitting scale-invariance across subjects between two images (see 469 below); (2) Context (two images extracted from either Within- or Across-chapter); (3) 
TMS stimulation (TMS-precuneus vs. TMS-vertex, see below).

Selection of 60 levels of temporal distances (TDs). In order to maximize the range of all TDs, we first selected the second longest chapter of the video game and determined the longest TD $(L)$, while ensuring the shortest TD to be longer than 30 frames. The 60 TD levels were selected according to this function,

$$
T D_{n}=L *\left(\frac{n}{60}\right)^{1.5}
$$

where $L$ denotes duration of the second longest chapter of the video game in each experimental session, $n$ denotes TD level, and value of $T D_{n}$ were rounded to the nearest integer using the "round" function in MATLAB. Note that the actual TDs were different across subjects, but since we applied a power function, the scale was thus rendered invariant (22). Image-pairs extraction from each of the chapters were independently conducted across subjects. The numbers of images-pairs extracted from each of the chapters were approximately equal within-subjects.

Transcranial magnetic stimulation. TMS procedure and protocol. TMS were applied using a 70 mm Double Air Film Coil connected to a Magstim Rapid2 (The Magstim Company, Ltd., Whitland, UK). In order to localize the target brain regions precisely, we obtained individual anatomical T1-weighted magnetic resonance images and then imported them into BrainSight (Rogue Research Inc., Montreal, Canada) for stereotaxic registration of the TMS coil with the participants' brain. The position of the coil and the subject's head were co-registered with BrainSight, and monitored using a Polaris Optical Tracking System (Northern Digital, Waterloo, Canada) during TMS. Positional 

and were superimposed onto the reconstructed three-dimensional MRI images of the subject using the BrainSight. The center of the coil was continuously monitored to be directly over the site of interest. For all sites (vertex, precuneus, and motor areas for measuring active motor threshold), the TMS coil was held tangential to the surface of the skull and was placed in a rostro-caudal direction. An adjustable frame was used to hold the TMS coil firmly in place, while the participants rested their heads on the chin rest. Head movements were monitored constantly by BrainSight and were negligible. delivered over the motor cortex necessary to elicit visible twitches of the right index finger in at least 5 out of 10 consecutive pulses. The location used to determine the active motor threshold was identified with a single pulse of TMS over the motor cortex at the left hemisphere. The TMS coil was systematically moved until the optimal cortical site was located to induce the largest and most reliable motor response; this stimulus output was then recorded. The TMS intensity was then calibrated at $110 \%$ of individual active motor threshold (stimulator output: $75.2 \pm 6.9 \%$, mean \pm se, range from 63\% to 88\%, S1 Table). In Experimental Session 1 and 2, the TMS was applied at a low-frequency rate of $1 \mathrm{~Hz}$ with an uninterrupted duration of $20 \mathrm{~min}$.

511 (MNI x, $\mathrm{y}, \mathrm{z}=6,-70,44)$, whereas the control stimulation was delivered to the vertex. right pre-auricular, and of the same distance to the nasion and the inion. Due to the 
514 folding of the two cerebral hemispheres, the stimulated vertex site lies at a considerable

515 distance from the TMS coil, thereby diminishing the effectiveness of the magnetic

516 pulses. Stimulating the vertex is not known to produce any memory task-relevant

517 effects and deemed as a reliable control site. Stimulation magnitude and protocols in

518 the present study were comparable to those used in similar studies that are robust to

519 produce significant memory-related changes by targeting at the precuneus (46-48) or

520 lateral parietal cortices $(31,32)$. Immediately after the end of the stimulation,

521 participants performed four runs of Temporal Order Judgment task in the MRI scanner

522 (delay period between the end of TMS and the beginning of MRI: $M_{\text {precuneus }}=15.29$ $\left.\min , M_{\text {vertex }}=20.76 \min , t(16)=-0.87, P=0.4\right)$.

MRI data acquisition and preprocessing. Data acquisition. All the participants were sagittal slices, $0.9 \mathrm{~mm}$ thickness, voxel size $=1 \times 1 \times 1 \mathrm{~mm}$ ). 

analyses (49). Data were analyzed using general linear models and representational

543 similarity analyses as described below with a high-pass filter cutoff of $256 \mathrm{~s}$ and autoregressive AR(1) model correction for auto-correlation.

Functional MRI data analysis. Parametric modulation analysis. First-level models

547 were performed on the fMRI data collected from the TMS-vertex session only (either

548 all the trials altogether or separately for Across-chapter vs. Within-chapter conditions).

549 In all of these models, each of the 240 trials was modeled with a canonical

550 hemodynamic response function as an event-related response with a duration of $5 \mathrm{~s}$.

For the TMS-vertex session as a whole (Across-chapter and Within-chapter trials

554 the TD pmod, we assigned the actual TD values at encoding as the modulatory

555 parameter, and used the polynomial function up to first order. Several regressors of no

556 interest were also included: 6 head movement regressors and 1 missing trial regressor

557 (i.e., no-response trials; number of missing trials of Across-chapter condition: $5.65 \pm$ 
6.96, of Within-chapter condition: $5.29 \pm 6.8 ; \mathrm{n}=17$, mean $\pm \mathrm{sd}$ ) and the run mean.

The purpose of this analysis was to test for any linear TD-dependent modulation of signal intensity in the brain between the TD between the two images at encoding and

561 the brain activity during TOJ retrieval of the same events. For the TD + RT pmod, we aimed to identify the voxels whose activities changed as a function of TD after the removal of the influence of reaction times. Each subjects' RTs corresponding to each TD level were entered as the modulatory parameter, together with the regressors of no interest as above. pmod analyses with identical sets of regressors as described above (namely, TD; TD + RT). We looked for changes in brain responses as a linear function of the regressor of interest (i.e., TD). Maps were created by multiple regression analyses between the observed signals and regressors. The contrast maps from the first-level model of

571 parametric analyses were taken for second-level group analyses and entered into one572 sample $t$-tests. The group analyses were performed for each contrast using a random 573 effects model(50). The statistical threshold was set at $p<0.05$ (FWE corrected) at 574 cluster level and $p<0.001$ at an uncorrected peak level according to the SPM12 575 standard procedure. The activation cluster locations were indicated by the peak voxels 576 on the normalized structural images and labeled using the nomenclature of Talairach 577 and Tournoux (1988) (51).

579 conducted using the RSA toolbox (http://www.mrc-cbu.cam.ac.uk/methods-and- 
resources/toolboxes/) on the fMRI data following realignment and normalization, but

TD level was modeled with a separate regressor and was contrasted to produce a Tstatistic map (spmT maps), creating 120 statistical maps in total (Across- vs. Withinradius of $9 \mathrm{~mm}$ (93 voxels, volume $=2,511 \mathrm{~mm}^{3}$ ) were extracted from the brain volume and then the data (i.e., signal intensity) for the 60 TD levels were Person productmoment $(1-r)$ correlated with every other level to generate a representational dissimilarity matrix (RDM), reflecting the between-condition dissimilarity of BOLD signal response. These neural RDMs were then Spearman-rank correlated with a set of

593 candidate RDMs (see Fig 3F), reflecting different predictions of the information carried

594 by similarity structure of neural signal responses and generated correlational maps (rmaps). Finally, these $r$-maps were converted to z-maps using Fisher transformation. All the $z$-maps were then submitted to a group-level one-sample $t$-test to identify voxels in

597 which the similarity between the predicted RDM and observed neural RDM was greater

598 than zero. This allowed us to identify voxels in which information of TD at retrieval 599 might be represented (see S3 Fig). The statistical threshold was set as identical to those employed in the univariate analysis, which was at $p<0.05$ (FWE corrected) at cluster 601 level and $p<0.001$ at an uncorrected peak level. 

applied a LOSO approach to create functional ROIs to avoid statistical bias (52). For instance, in order to identify an ROI (i.e., conjunction mask in Fig 3D) for Subj01, we estimated the contrast using a one-sample $t$-test on the whole-brain searchlight z-maps

We set the same threshold reported above to extract clusters from these two statistical maps. We then overlaid the two resultant maps and extracted a conjunction region different ROIs, which provided statistically independent regions to extract values for and occipital cortex) of AAL template (53) were created as masks. We extracted and averaged the similarity value within these masks for each subject for statistical tests. 
corresponding cells of the RDM.

Precuneus and temporal context memory

Model 2 (Situational Change RDM, $60 \times 60$ ): Since the temporal and spatial dimensions were closely inter-correlated. We checked whether the situational change might influence the neural patterns in those voxels that represent the TD information. We analyzed the subject-specific videos frame by frame and marked out the boundaries at which a situational location had changed (see illustration in S4 Fig). Then we computed the numbers of situational changes contained in each of the paired images and then computed the differences with and among every other conditions producing $60 \times 59 / 2$ values, which were then assigned to the corresponding cells of the RDM.

Model 3 (RGB-cross-correlation RDM, 60 × 60), Model 4 (RGB-intensity RDM, $60 \times 60)$ and Model 5 (RGB-histogram RDM, $60 \times 60$ ) considered the perceptual characteristics of the images used in TOJ. For Model 3, the similarity measure was based on the cross-correlation value between two images (image of size $1920 \times 1080$ ) for the three color channels (red, green, and blue; RGB). For every pair of images in each of the three color channels (RGB), we computed the cross-correlation coefficients between the pair. This is a measure of the displacement of one image relative to the other; the larger the cross-correlation coefficient (which ranges between -1 and 1), the more similar the two images was. We then computed the differences with and among every other conditions producing $60 \times 59 / 2$ values, which were then assigned to the corresponding cells of the RDM. For Model 4, we computed the pixel-wise difference between pair images for the three color channels (RGB). The computed difference is useful when the compared images are taken from a stationary camera with infinitesimal 

the absolute difference between the corresponding pixels in the image pair is non-zero, or a value of 0 otherwise. A single value is generated for each of the three color channels by summing all the output pixel values (either 0 or 1 ). We averaged the sum of difference for all three color-channels for the intensity value of each pair of images and then computed the differences with and among every other conditions producing $60 \times$ 59/2 values, which were then assigned to the corresponding cells of the RDM. For Model 5, we constructed color histograms for image pairs and computed the Sum-ofSquare-Difference (SSD) error between them for the three color channels (RGB). For each color channel the intensity values range from 0 to 255 (i.e., 256 bins), we first computed the total number of pixels at each intensity value and then computed the SSD for all 256 bins for each image pair. The smaller the value of the SSD, the more similar the two images (image pair) was. We then computed the differences with and among every other conditions producing $60 \times 59 / 2$ values, which were then assigned to the corresponding cells of the RDM. In contrast to model 4, this approach does not require corresponding pixels in the image pair to be the same, but rather measures the existence of pixel intensity in both images. Overall, the three perceptual-similarity models (3, 4 and 5) look at different similarity measures and they complement each other; thus any difference in the appearance of the two images irrespective of the temporal distance, could be accounted for by at least one of the three models. For any two similar images, the RGB-intensity RDM results in a very small value, thus the corresponding pixels are

667 virtually the same for the entire image. The RGB-histogram will also result in a small 
value as the image pairs will have the same histogram bins. The RGB-cross-correlation value will be close to 1 , signifying the similarity in the images. When subsections of a scene are visible in both images with varied brightness, the RGB-cross-correlation value will still be closer to 1 but with a very high RGB-intensity RDM value.

Model 6 (Scale Invariant Feature Transform (SIFT) RDM, $60 \times 60$ ), Model 7 (Speeded Up Robust Features (SURF) RDM, $60 \times 60$ ) and Model 8 (Histogram of Oriented Gradients $(\mathrm{HOG}), 60 \times 60$ ): For model 6, SIFT transform images into scaleinvariant vectors which encoded interest points. For any pair of images to be compared, a computational search over selected scales and image locations is conducted using Difference-of-Gaussian (DoF) to identify potential interest points that are invariant to scale and orientation in each image. Interest points from each image are stabilized using Taylor series expansion of scale space to get a more accurate location of extrema. This is followed by the construction of an orientation histogram to achieve invariance to rotation. Key-points between the two images are then matched by identifying their nearest neighbors using minimum Euclidean distance between the invariant descriptor vectors extracted from each image. For model 7, rather than the use of DoF in SIFT (26) as an approximation of Laplacian-of-Gaussian (LoG), SURF (27) uses Box Filter which is calculated in parallel using integral images (54) to approximate LoG. Wavelet responses in both horizontal and vertical directions are used to assign orientation in SURF. Like SIFT, the first step in SURF consists of fixing a reproducible orientation based on information from a circular region around the interest point (27). A descriptor vector is generated around the interest point using integral image, which is compared 

nor Aminoff et al (25) but it is robust and much faster to compute than SIFT. However, ImageNet 2012 to find visual features to perform their categorization. To remove the time for training a supervised network like CNN which uses SIFT/SURF features in it layers, our dissimilarity measures are based on the features extracted solely from the input images with no prior training. For model 8, in the case of the HOG feature descriptor, we constructed a histogram of directions of gradient over fixed sized grid across the entire image. A vector is generated from each grid cell and correlated with HOG features from another image. It is worth noting that gradients or derivatives of $\mathrm{x}$ and y (location) in an image are useful for interest point localization because they have higher values around edges and corners, which hold more information about objects. Model $9(\mathrm{RT}$ RDM, $60 \times 60)$ and Model $10($ ACC RDM, $60 \times 60)$ were computed based on the behavioral performance of reaction times and percentage correct ( 0 or 1$)$ chapter or Across-chapter alone) and computed the differences with and among every other TD levels producing $60 \times 59 / 2$ values, which were then assigned to the 


\section{Supporting Information}

A
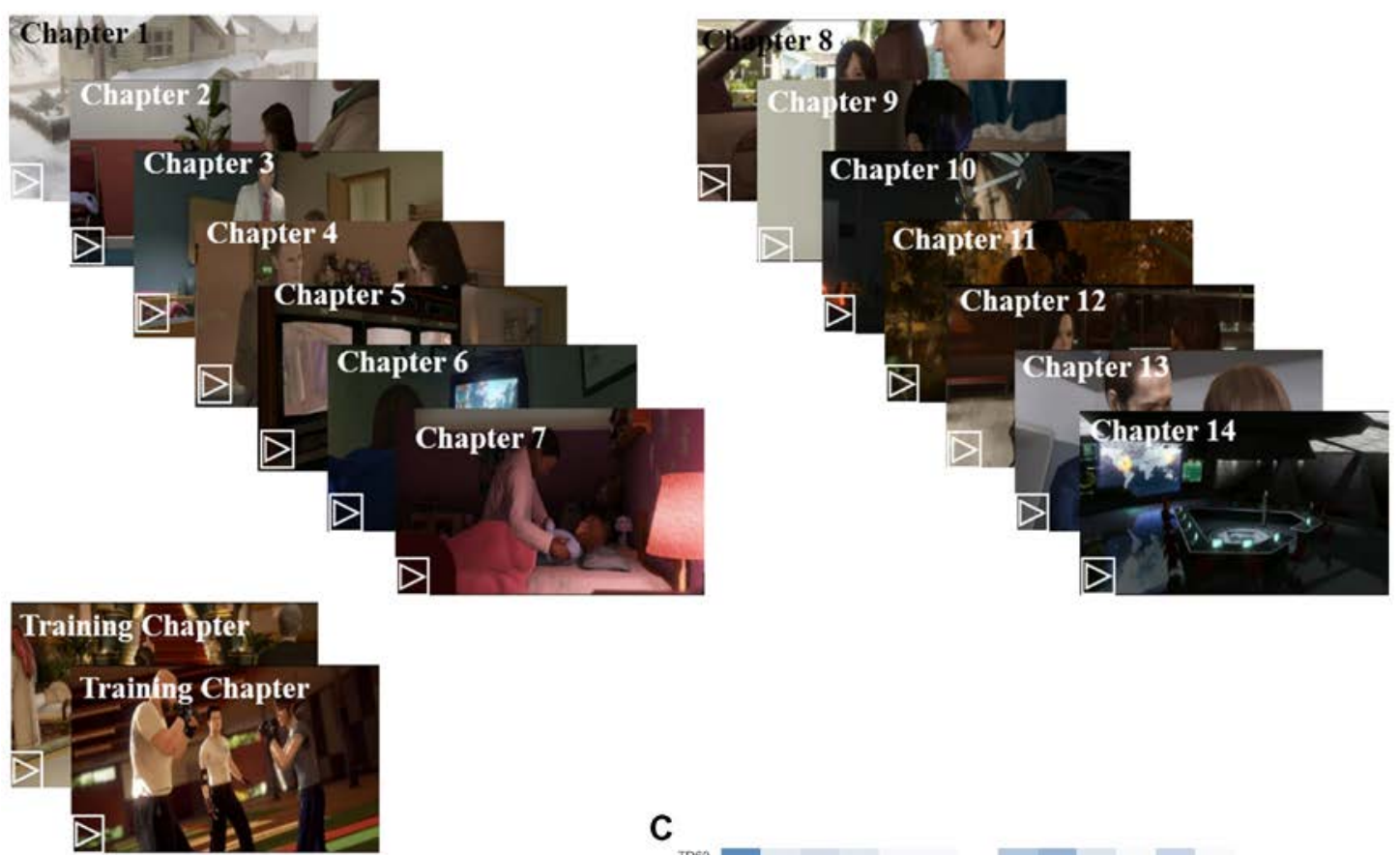

Precuneus and temporal context memory

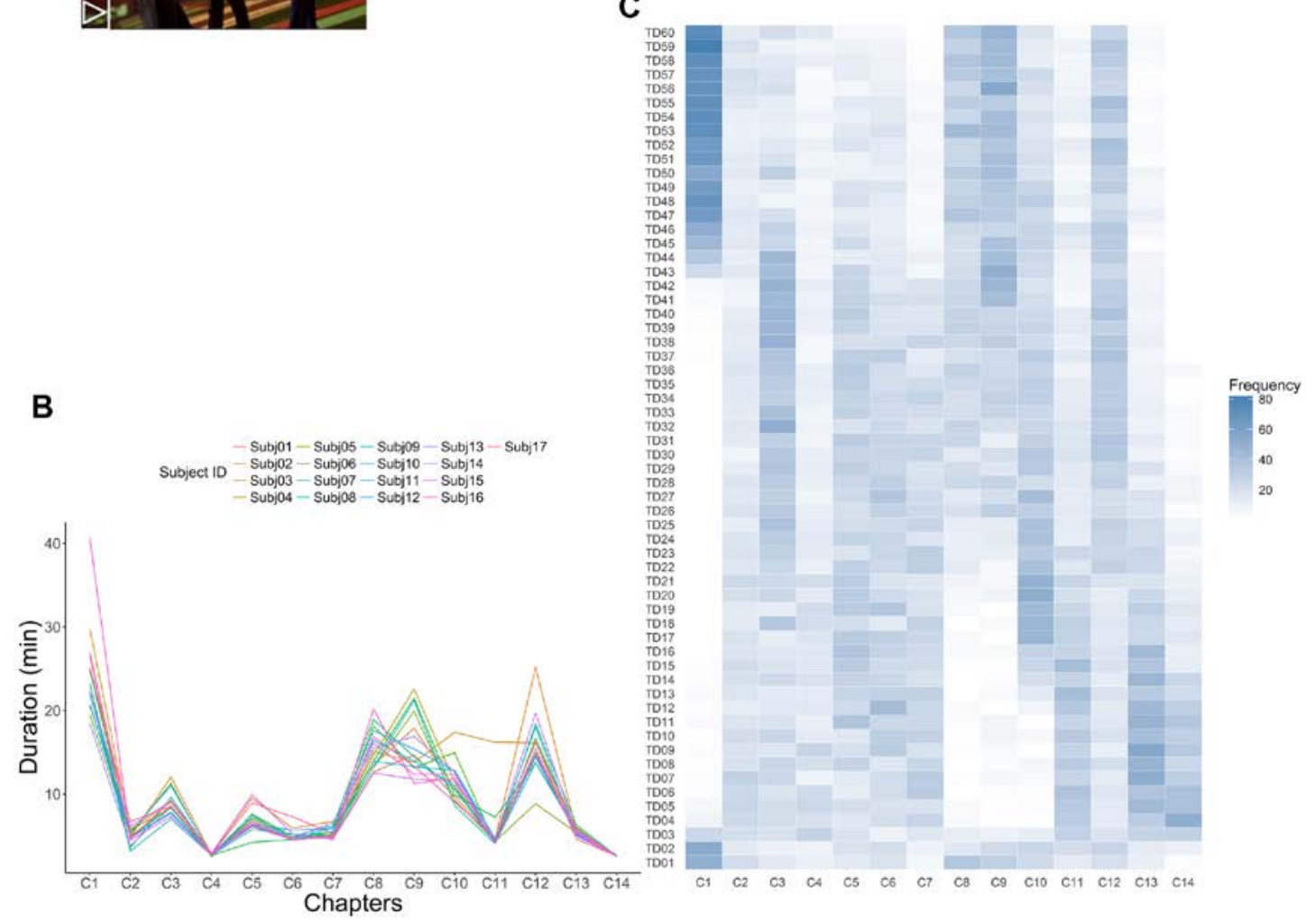

714 S1 Fig. Stimuli. (A) 14 different chapters were used: seven in each of two within-

715 subjects TMS sessions (see also S1 Table). (B) Playing durations varied across chapters and subjects. (C) Intensity map showed that the numbers of trials extracted from the 14 
717 game chapters separately for the 60 TD levels across subjects. The numbers of image

718 pairs extracted for the TOJ task were approximately equal across the chapters, except

719 that longer TD are more likely to be extracted from the longest chapter (e.g., C1).

720 


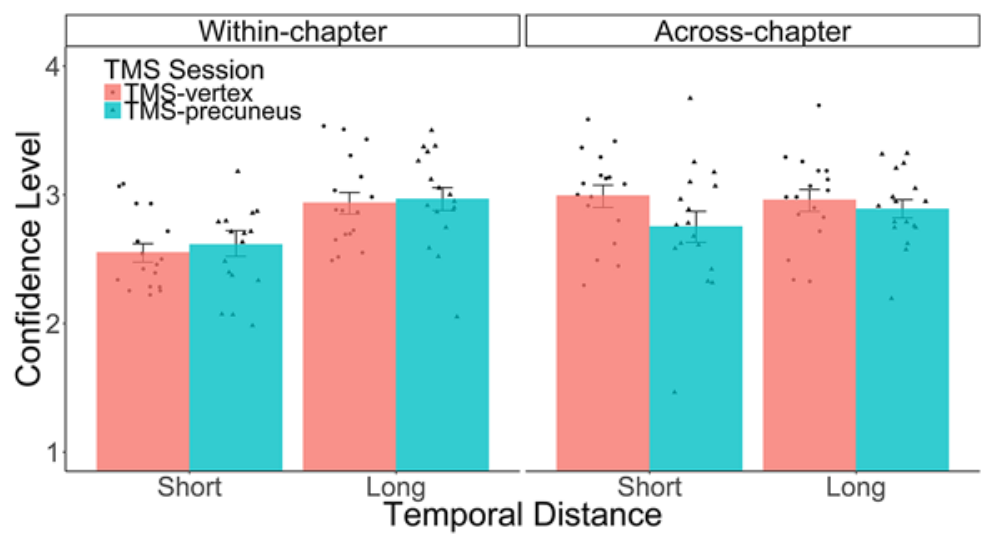

S2 Fig. Behavioral results of confidence level. TMS did not result in differences in $\left.=19.12, P<0.001, \eta^{2}=4.52 \%\right)$, which was driven by significant differences in

725 confidence level between short and long TD in Within-chapter condition $\left(t_{(33)}=6.76\right.$, $P<0.001)$, but not in Across-chapter condition $\left(t_{(33)}=1.17, P=0.250\right)$. Dots indicate behavioral performance per subject. Error bars denote the SEM over subjects. 


\section{using TD RDM to perform searchlight RSA as an example}

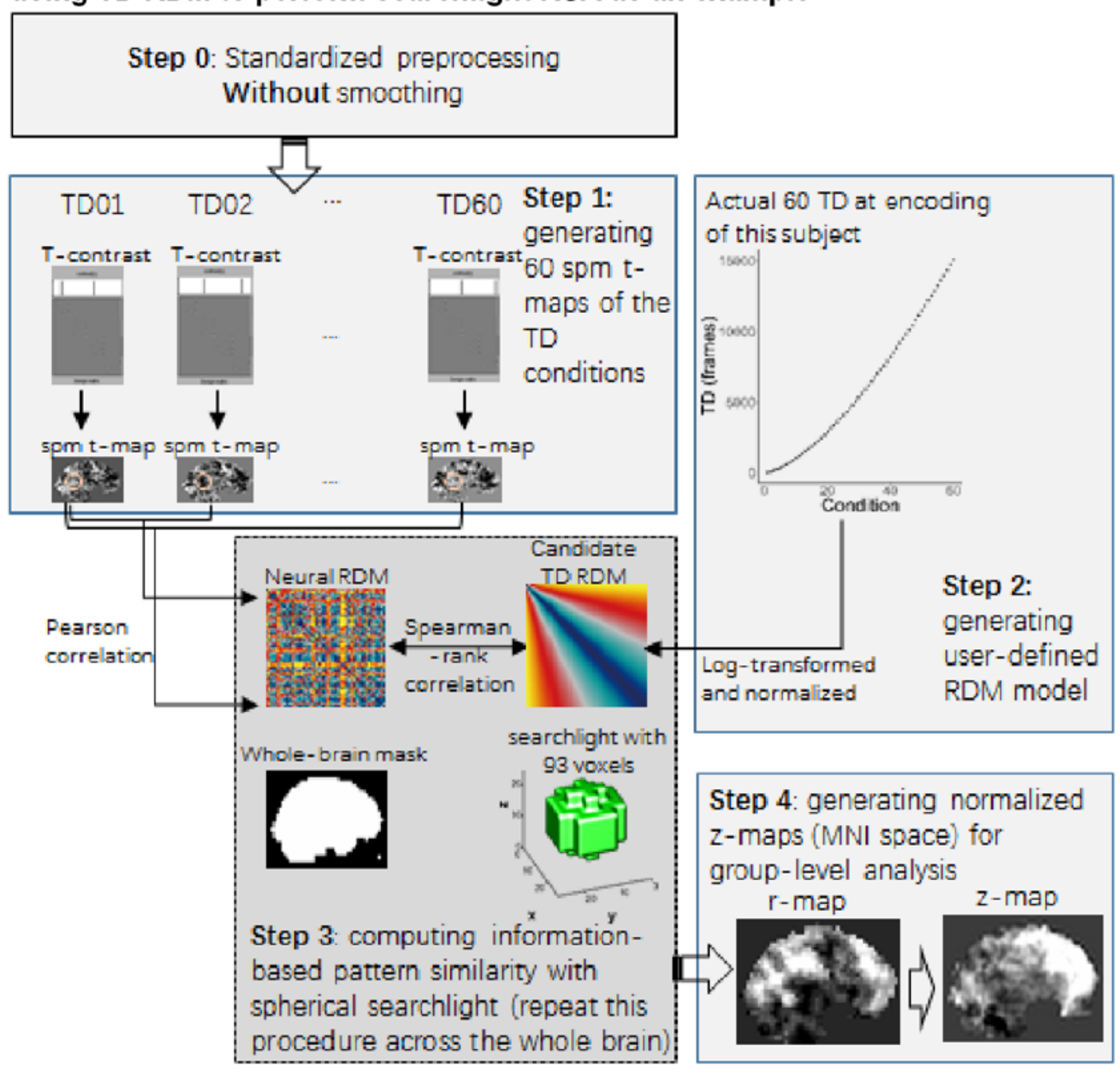

S3 Fig. Pipeline of RSA analysis. Using one model (TD RDM) to perform the 
A
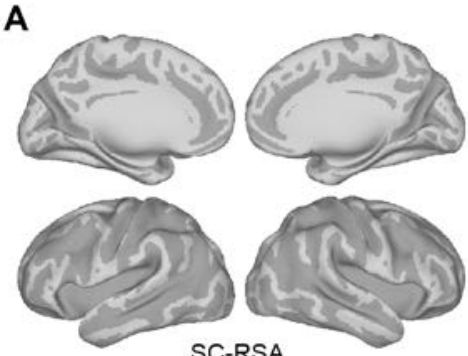

D
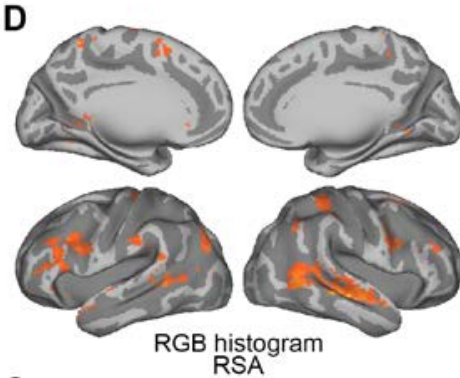

G
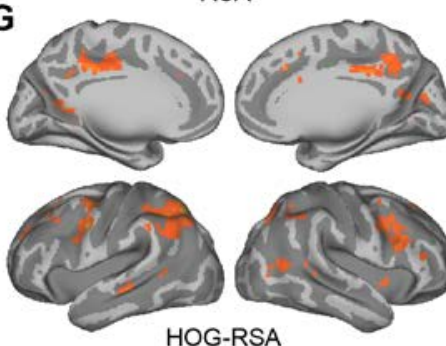

$\mathbf{J}$
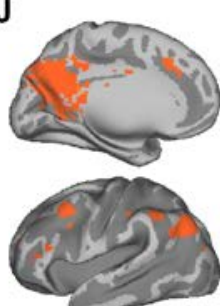

partial TD-RSA
B

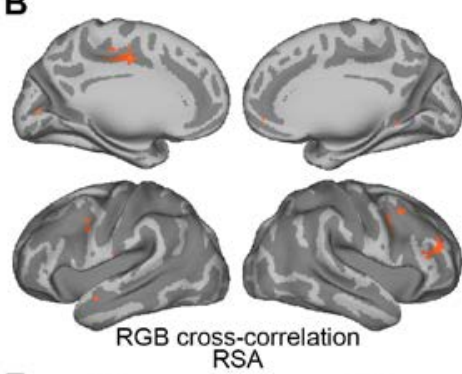

E
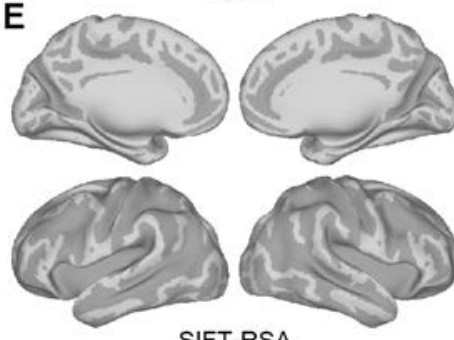

SIFT-RSA

H
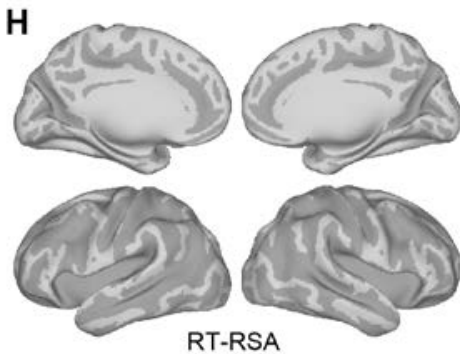

RT-RSA
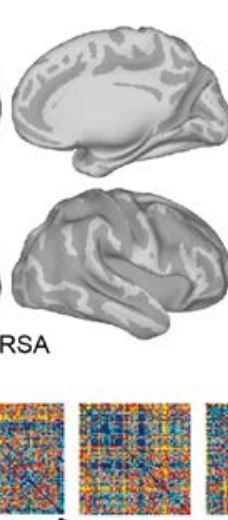

象
C

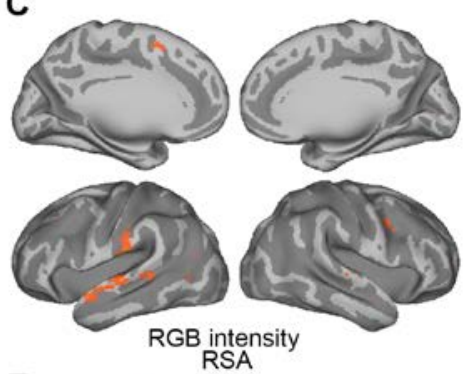

$\mathbf{F}$
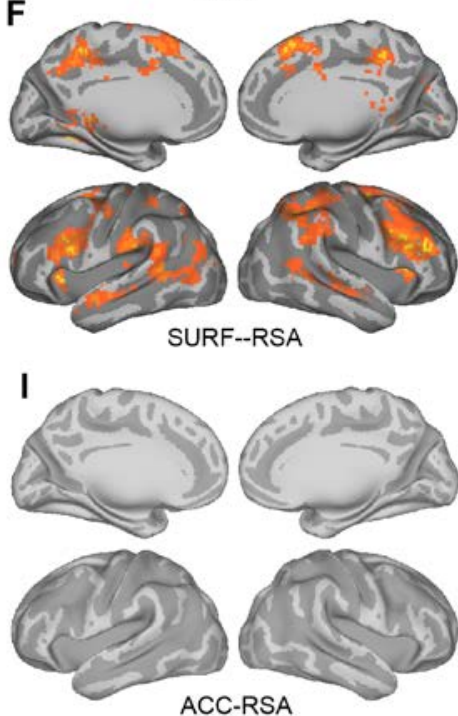

mesces

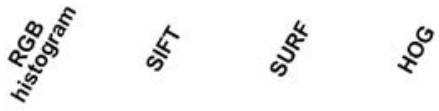

S4 Fig. RSA searchlight results for TMS-vertex sessions using candidate RDMs.

(A) Situational Change RDM (see S5 Fig). (B) RGB cross-correlation RDM. (C) RGB- 


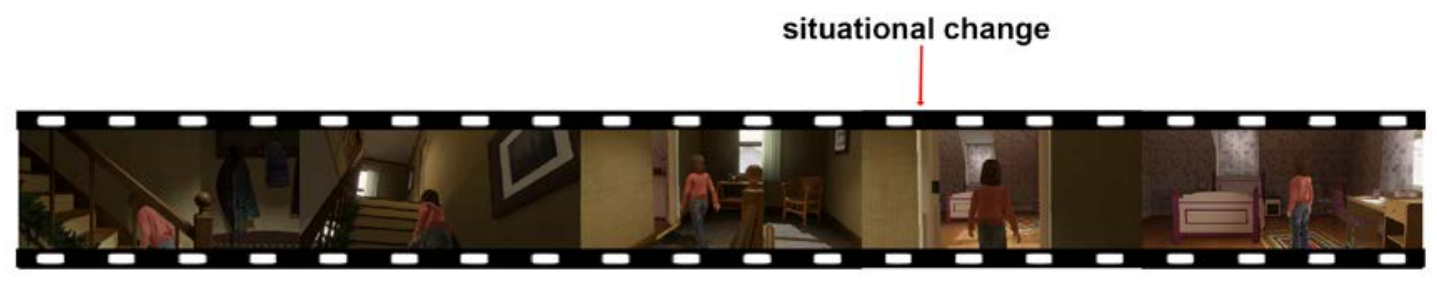

744 S5 Fig. An excerpt of a subject-specific video. An excerpt of a subject-specific video

745 illustrates how situational changes were defined frame-by-frame for Situational Change

746 RDMs and pmod analyses. Noting that time and space in our task were partially

747 correlated, we quantified subject-specific situational changes in terms of the number of

748 locations each participant had traversed in the video (i.e., spatial displacement

749 embedded in the paired images presented in TOJ task). 
A

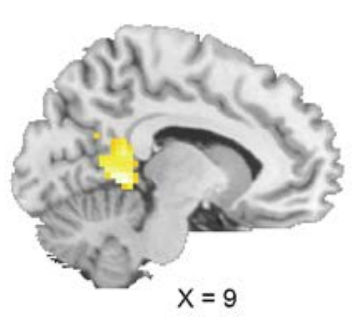

B

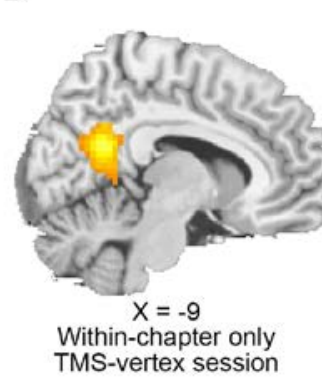

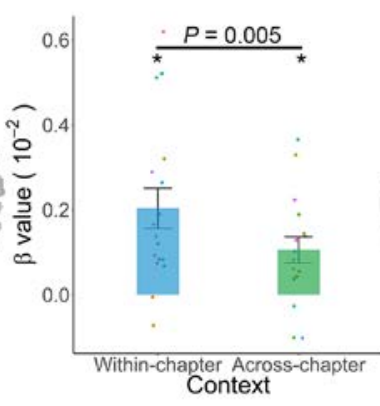

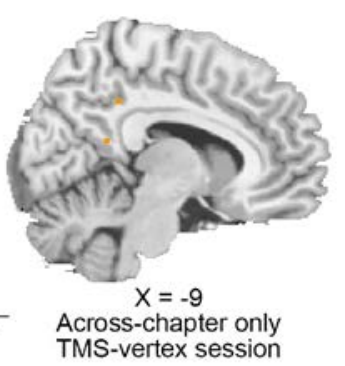

C
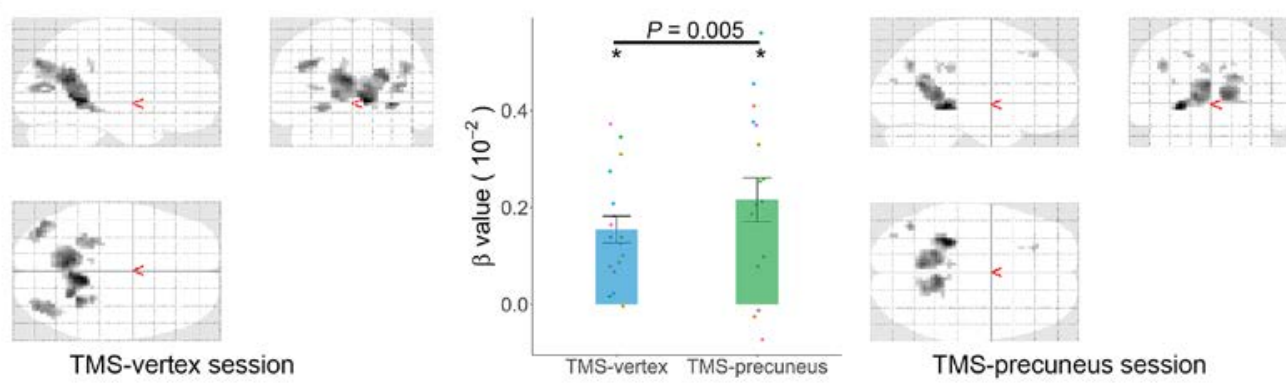

S6 Fig. Pmod analyses for TMS-vertex session and TMS-precuneus session. (A)

Temporal distance (TD) and reaction time (RT) as modulatory regressors for TMS-

vertex session (Within-chapter and Across-chapter trials collapsed). Having removed

the influence of RT, neural activity in the precuneus/retrosplenial cortex and posterior hippocampus still increased with TD. (B) TD and RT as modulatory regressors separately for Within-chapter (left) and Across-chapter (right) conditions in the TMSvertex session. After having removed the influence of RT, neural activity in the precuneus, extending into the retrosplenial cortex and posterior cingulate cortex, remained linearly associated with TD in Within-chapter condition. The $\beta$ values extracted from the cluster was also higher in the Within-chapter condition than in the Across-chapter condition $(P=0.005)$. (C) In contrast to the removal of the multivoxel representation, TMS to the precuneus did not impact on the activation intensity in the precuneus and retrosplenial cortex (right). Moreover, activation in these clusters 
bioRxiv preprint doi: https://doi.org/10.1101/249904; this version posted October 5, 2018. The copyright holder for this preprint (which was not certified by peer review) is the author/funder, who has granted bioRxiv a license to display the preprint in perpetuity. It is made available under aCC-BY 4.0 International license.

Precuneus and temporal context memory

766 stimulation (middle panel). Activation maps are displayed at $P_{\text {uncorrected }}<0.001$.

767 
bioRxiv preprint doi: https://doi org/101101/249904; this version posted October 5,2018 . The copyright holder for this preprint (which was not certified by peer review) is the author/funder, who has granted bioRxiv a license to display the preprint in perpetuity. It is made available under aCC-BY 4.0 International license.

Precuneus and temporal context memory

A

B
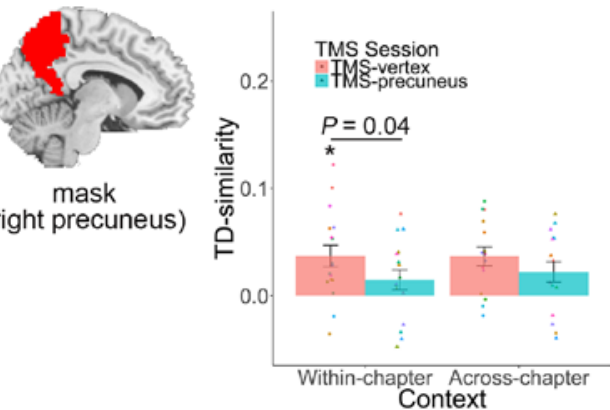

C
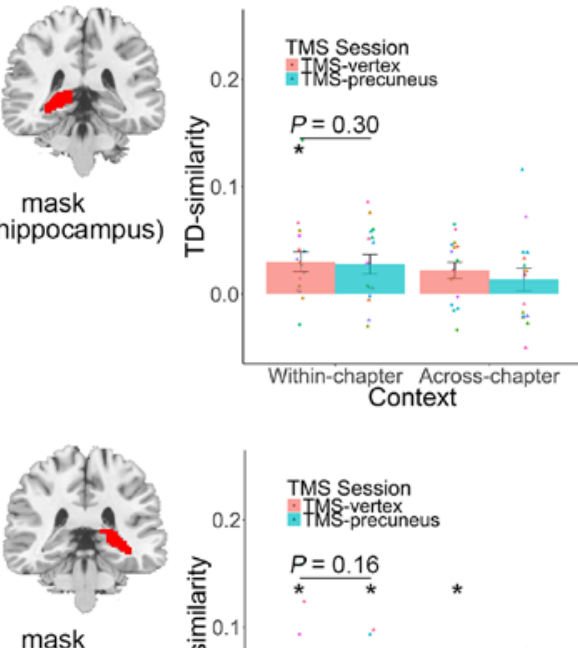

(right hippocampus)

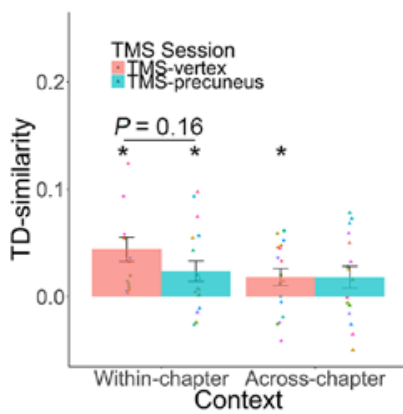

D
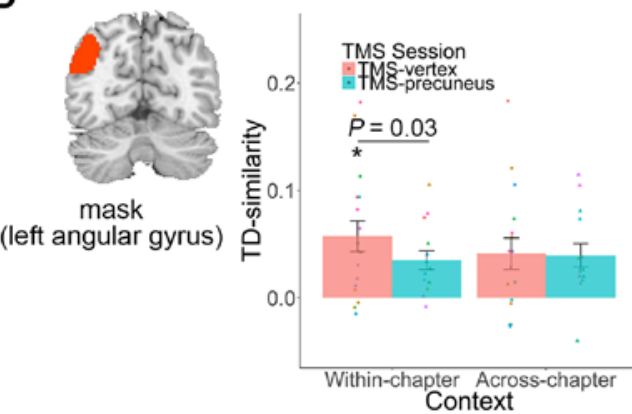

E

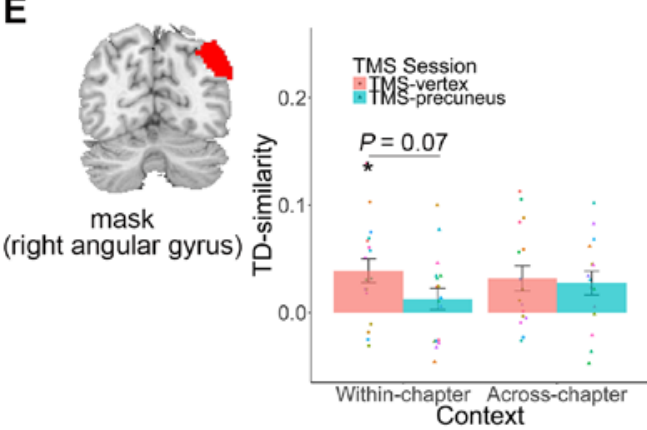

$\mathbf{F}$
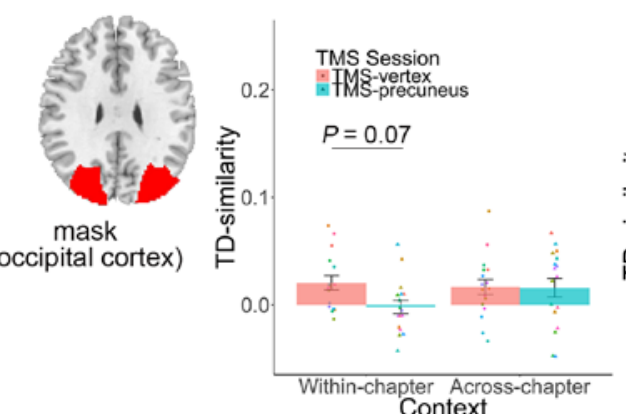
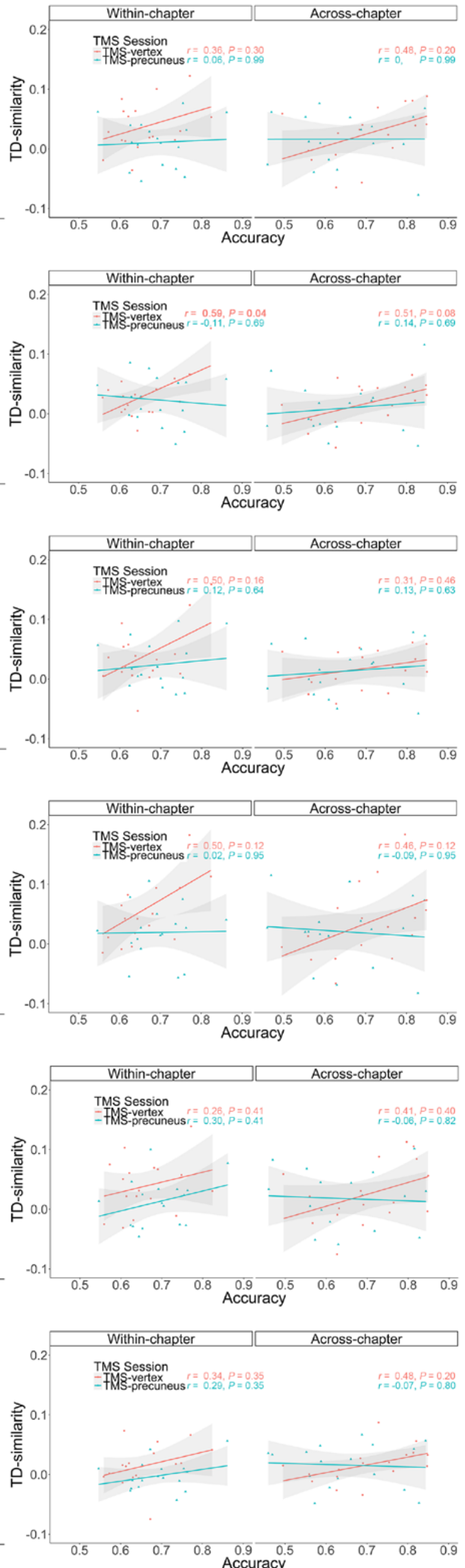
S7 Fig. TD-similarity indices in six anatomical ROIs (A: right precuneus, B-C:

771 separately for Within-chapter and Across-chapter conditions, in both TMS sessions,

772 and their correlation with individual subjects TOJ accuracy. ${ }^{*} P<0.05$, FDR-corrected,

773 one-sample $t$-test against zero. Horizontal lines indicate paired $t$-test between two bars

774 and $P$ values are FDR-corrected.

775 
A
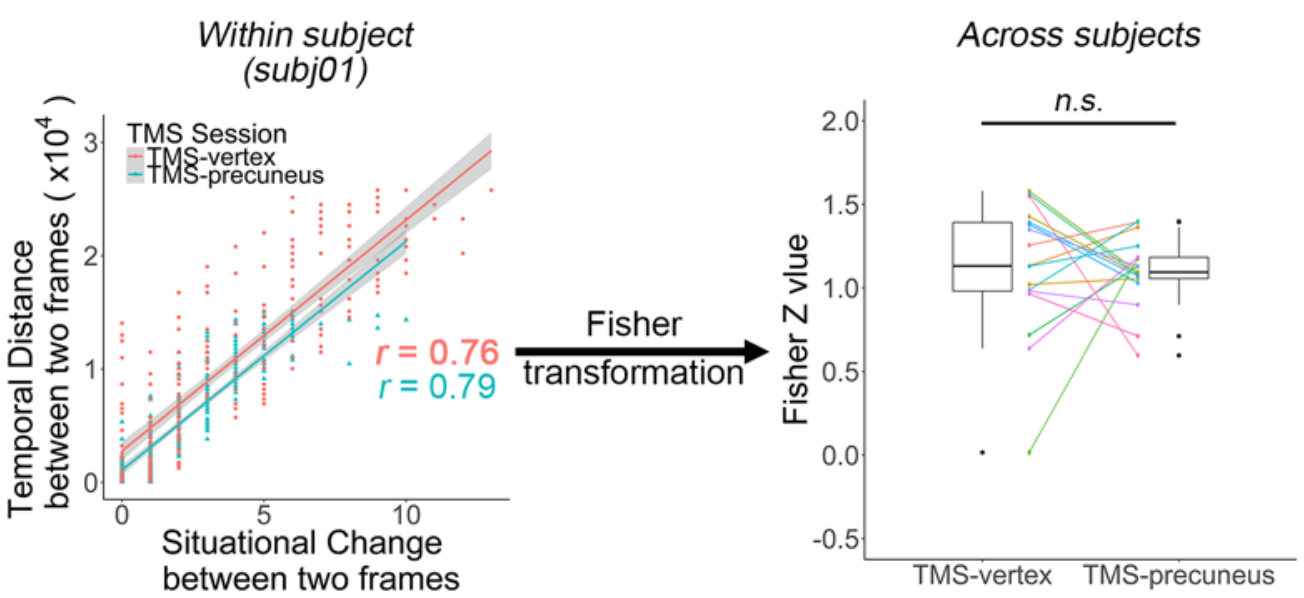

B

Across subjects
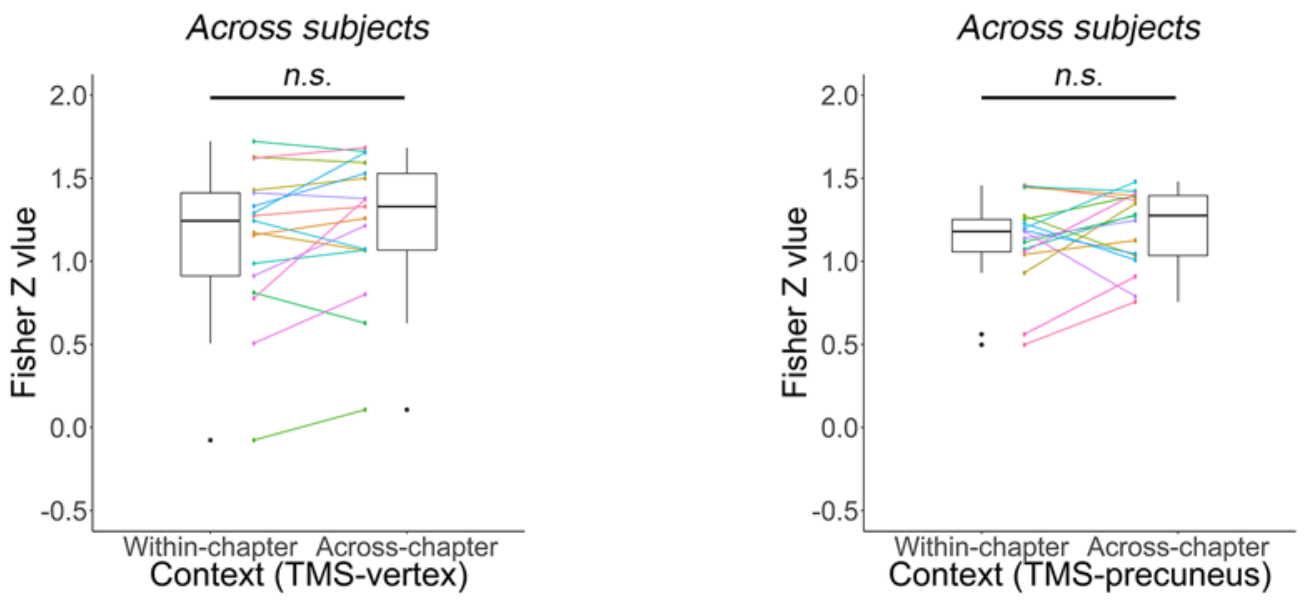

S8 Fig. Correlation coefficients. Correlation coefficients were very high between

zero (TMS-vertex session: mean $\pm \mathrm{se}=1.12 \pm 0.1, t_{16}=11.52, P=3.71 \times 10^{-9}$; TMS-

precuneus session: mean $\left.\pm \mathrm{se}=1.10 \pm 0.05, t_{16}=21.08, P=4.24 \times 10^{-13}\right)$. No significant 
787 Fisher $z$ values for all participants in the Across-chapter and Within-chapter conditions

788 of two TMS sessions separately. $Z$ values are significantly greater than zero in each

789 conditions $(P<0.01)$. No significant differences exist between the two sessions $(P>$

790 0.05). n.s. denotes not significant.

791 (TIF)

792 
S1 Table. Descriptive statistics of TMS motor threshold and full counter-

balancing for gameplay chapters by TMS sites.

795

\begin{tabular}{|c|c|c|c|c|c|c|c|c|}
\hline \multirow[b]{2}{*}{ ID } & \multirow{2}{*}{$\begin{array}{c}\text { Motor } \\
\text { thresh } \\
\text { old }\end{array}$} & \multicolumn{3}{|c|}{ Session 1} & \multirow{2}{*}{$\begin{array}{l}\text { Delay } \\
\text { (days) }\end{array}$} & \multicolumn{3}{|c|}{ Session 2} \\
\hline & & $\begin{array}{l}\text { Chapters } \\
\text { (Playing) }\end{array}$ & $\begin{array}{l}\text { Duration } \\
\text { (min) }\end{array}$ & TMS Site & & $\begin{array}{l}\text { Chapters } \\
\text { (Playing) }\end{array}$ & $\begin{array}{l}\text { Duration } \\
\text { (min) }\end{array}$ & TMS Site \\
\hline Subj01 & $66 \%$ & Chap $1 \sim 7$ & 89.39 & Precuneus & 9 & Chap $8 \sim 14$ & 35.19 & Vertex \\
\hline Subj02 & $67 \%$ & Chap $1 \sim 7$ & 102.70 & Vertex & 6 & Chap $8 \sim 14$ & 44.46 & Precuneus \\
\hline Subj03 & $80 \%$ & Chap $8 \sim 14$ & 35.53 & Vertex & 9 & Chap $1 \sim 7$ & 114.21 & Precuneus \\
\hline Subj04 & $58 \%$ & Chap $8 \sim 14$ & 36.41 & Vertex & 2 & Chap $1 \sim 7$ & 94.70 & Precuneus \\
\hline Subj05 & $65 \%$ & Chap $1 \sim 7$ & 80.50 & Precuneus & 8 & Chap $8 \sim 14$ & 35.31 & Vertex \\
\hline Subj06 & $67 \%$ & Chap $1 \sim 7$ & 95.05 & Vertex & 3 & Chap $8 \sim 14$ & 39.63 & Precuneus \\
\hline Subj07 & $72 \%$ & Chap $8 \sim 14$ & 32.41 & Vertex & 4 & Chap $1 \sim 7$ & 97.06 & Precuneus \\
\hline Subj08 & $73 \%$ & Chap $1 \sim 7$ & 96.08 & Precuneus & 8 & Chap $8 \sim 14$ & 37.03 & Vertex \\
\hline Subj09 & $69 \%$ & Chap $1 \sim 7$ & 88.81 & Vertex & 7 & Chap $8 \sim 14$ & 31.46 & Precuneus \\
\hline Subj10 & $80 \%$ & Chap $8 \sim 14$ & 35.69 & Precuneus & 7 & Chap $1 \sim 7$ & 89.79 & Vertex \\
\hline Subj11 & $65 \%$ & Chap $8 \sim 14$ & 38.77 & Vertex & 26 & Chap $1 \sim 7$ & 86.02 & Precuneus \\
\hline Subj12 & $71 \%$ & Chap $1 \sim 7$ & 90.56 & Precuneus & 16 & Chap $8 \sim 14$ & 35.89 & Vertex \\
\hline Subj13 & $68 \%$ & Chap $8 \sim 14$ & 35.66 & Vertex & 7 & Chap $1 \sim 7$ & 87.98 & Precuneus \\
\hline Subj14 & $73 \%$ & Chap $1 \sim 7$ & 87.94 & Precuneus & 7 & Chap $8 \sim 14$ & 40.56 & Vertex \\
\hline Subj15 & $57 \%$ & Chap $8 \sim 14$ & 32.72 & Precuneus & 5 & Chap $1 \sim 7$ & 91.46 & Vertex \\
\hline Subj16 & $69 \%$ & Chap $8 \sim 14$ & 35.44 & Vertex & 5 & Chap $1 \sim 7$ & 108.49 & Precuneus \\
\hline Subj17 & $61 \%$ & Chap $1 \sim 7$ & 91.10 & Precuneus & 6 & Chap $8 \sim 14$ & 42.13 & Vertex \\
\hline
\end{tabular}


S2 Table. Brain representation associated with TD RDM in TMS-vertex session

(see Fig 3B).

800

\begin{tabular}{|c|c|c|c|c|c|c|}
\hline \multicolumn{2}{|c|}{ Cluster } & \multicolumn{3}{|c|}{ peak } & \multirow{2}{*}{$\%$ Cluster } & \multirow{2}{*}{ Brain regions } \\
\hline $\mathbf{k}$ & p-FWE & $\mathbf{X}$ & $\mathbf{y}$ & $\mathbf{z}$ & & \\
\hline \multirow[t]{30}{*}{7554} & $<.001$ & 30 & 21 & 38 & 5.41 & Precuneus_L \\
\hline & & & & & 5.32 & Precuneus_R \\
\hline & & & & & 4.38 & Frontal_Mid_2_R \\
\hline & & & & & 3.34 & Angular_R \\
\hline & & & & & 3.24 & Parietal_Inf_L \\
\hline & & & & & 3.15 & Frontal_Inf_Tri_R \\
\hline & & & & & 2.99 & Parietal_Inf_R \\
\hline & & & & & 2.91 & Frontal_Mid_2_L \\
\hline & & & & & 2.83 & Frontal_Inf_Tri_L \\
\hline & & & & & 2.74 & Angular_L \\
\hline & & & & & 2.73 & SupraMarginal_R \\
\hline & & & & & 2.73 & Frontal_Inf_Oper_R \\
\hline & & & & & 2.65 & Frontal_Sup_2_R \\
\hline & & & & & 2.61 & Cuneus_L \\
\hline & & & & & 2.58 & Cingulate_Mid_R \\
\hline & & & & & 2.57 & Postcentral_R \\
\hline & & & & & 2.55 & Temporal_Mid_R \\
\hline & & & & & 2.26 & Calcarine_L \\
\hline & & & & & 2.22 & Cuneus_R \\
\hline & & & & & 1.87 & Precentral_R \\
\hline & & & & & 1.85 & Occipital_Mid_R \\
\hline & & & & & 1.62 & Calcarine_R \\
\hline & & & & & 1.62 & Frontal_Sup_Medial_R \\
\hline & & & & & 1.56 & Cingulate_Mid_L \\
\hline & & & & & 1.52 & Occipital_Sup_R \\
\hline & & & & & 1.47 & Frontal_Sup_Medial_L \\
\hline & & & & & 1.42 & Temporal_Sup_R \\
\hline & & & & & 1.22 & Occipital_Mid_L \\
\hline & & & & & 1.02 & Cingulate_Post_L \\
\hline & & & & & 1.01 & Precentral_L \\
\hline
\end{tabular}


in TMS-vertex session (see Fig 3C).

805

\begin{tabular}{|c|c|c|c|c|c|c|c|}
\hline \multirow[t]{2}{*}{ Regressor } & \multicolumn{2}{|c|}{ Cluster } & \multicolumn{4}{|l|}{ Voxel } & \multirow[t]{2}{*}{ Brain Regions } \\
\hline & $\mathbf{k}$ & $\begin{array}{l}\text { p- } \\
\text { FWE }\end{array}$ & $Z$ value & $\mathbf{x}$ & $\mathbf{Y}$ & $\mathbf{z}$ & \\
\hline \multirow[t]{9}{*}{$\mathrm{TD}$} & 555 & $<.001$ & 4.62 & 9 & -45 & -1 & Cerebelum_4_5_R \\
\hline & & & 4.35 & -6 & -57 & 20 & Precuneus_L \\
\hline & & & 4.27 & 6 & -54 & 5 & Vermis_4_5 \\
\hline & 116 & .002 & 3.87 & 36 & -81 & 14 & Occipital_Mid_L \\
\hline & & & 3.78 & 42 & -72 & 29 & Occipital_Mid_L \\
\hline & & & 3.75 & 36 & -69 & 11 & Occipital_Mid_R \\
\hline & $117^{\dagger}$ & .01 & 4.34 & 45 & -36 & 5 & Temporal_Sup_R \\
\hline & & & 4.17 & 54 & -36 & 5 & Temporal_Sup_R \\
\hline & & & 3.81 & 48 & -27 & -1 & Temporal_Sup_R \\
\hline
\end{tabular}

S1 Video. An excerpt of the gameplay video.

\section{Acknowledgements}

This research is sponsored by the National Natural Science Foundation of China 


\section{Author contributions}

821 Q.Y. designed and conducted the study, analyzed data, drafted and wrote the manuscript.

822 Y.H. discussed the results and commented on drafts. Y.K. advised on TMS protocol.

823 K.A. produced indices for RDM Models 4, 5, 6, 7 and 8. S.C.K. designed the study,

824 supervised the research, and wrote the manuscript.

825 Competing interests: The authors declare no competing interests.

826 Code availability. The codes used for the analyses are available upon request.

827 Data availability. The data reported in this study are available on

828 http://datadryad.org/review?doi=doi:10.5061/dryad.pj038. 
Precuneus and temporal context memory

\section{References}

1. Buhusi CV, Meck WH. What makes us tick? Functional and neural mechanisms of interval timing. Nat Rev Neurosci. 2005;6(10):755-65.

2. Meck WH, Penney TB, Pouthas V. Cortico-striatal representation of time in animals and humans. Curr Opin Neurobiol. 2008;18(2):145-52.

3. Mauk MD, Buonomano DV. The neural basis of temporal processing. Annu Rev Neurosci. 2004;27:307-40.

4. Leon MI, Shadlen MN. Representation of time by neurons in the posterior parietal cortex of the macaque. Neuron. 2003;38(2):317-27.

5. Jin DZ, Fujii N, Graybiel AM. Neural representation of time in cortico-basal ganglia circuits. Proc Natl Acad Sci U S A. 2009;106(45):19156-61.

6. Naya Y, Suzuki WA. Integrating What and When Across the Primate Medial Temporal Lobe. Science. 2011;333(6043):773-6.

7. Tsao A, Sugar J, Lu L, Wang C, Knierim JJ, Moser MB, et al. Integrating time from experience in the lateral entorhinal cortex. Nature. 2018.

8. McGaugh JL. Memory--a century of consolidation. Science. 2000;287(5451):248-51.

9. Ezzyat Y, Davachi L. Similarity breeds proximity: pattern similarity within and across contexts is related to later mnemonic judgments of temporal proximity. Neuron. 2014;81(5):1179-89.

10. Manns JR, Howard MW, Eichenbaum H. Gradual changes in hippocampal activity support remembering the order of events. Neuron. 2007;56(3):530-40.

11. Nielson DM, Smith TA, Sreekumar V, Dennis S, Sederberg PB. Human hippocampus represents space and time during retrieval of real-world memories. Proc Natl Acad Sci U S A. 2015;112(35):1107883.

12. Hsieh LT, Gruber MJ, Jenkins LJ, Ranganath C. Hippocampal activity patterns carry information about objects in temporal context. Neuron. 2014;81(5):1165-78.

13. Ranganath $\mathrm{C}$, Ritchey $\mathrm{M}$. Two cortical systems for memory-guided behaviour. Nat Rev Neurosci. 2012;13(10):713-26.

14. Baldassano C, Chen J, Zadbood A, Pillow JW, Hasson U, Norman KA. Discovering Event Structure in Continuous Narrative Perception and Memory. Neuron. 2017;95(3):709-21 e5.

15. Chen J, Leong YC, Honey CJ, Yong CH, Norman KA, Hasson U. Shared memories reveal shared structure in neural activity across individuals. Nat Neurosci. 2017;20(1):115-25.

16. Honey CJ, Thesen T, Donner TH, Silbert LJ, Carlson CE, Devinsky O, et al. Slow cortical dynamics and the accumulation of information over long timescales. Neuron. 2012;76(2):423-34.

17. Polyn SM, Norman KA, Kahana MJ. A context maintenance and retrieval model of organizational processes in free recall. Psychol Rev. 2009;116(1):129-56.

18. Kwok SC, Shallice T, Macaluso E. Functional anatomy of temporal organisation and domainspecificity of episodic memory retrieval. Neuropsychologia. 2012;50(12):2943-55.

19. St Jacques P, Rubin DC, LaBar KS, Cabeza R. The short and long of it: neural correlates of temporal-order memory for autobiographical events. J Cogn Neurosci. 2008;20(7):1327-41.

20. Richter FR, Cooper RA, Bays PM, Simons JS. Distinct neural mechanisms underlie the success, precision, and vividness of episodic memory. Elife. 2016;5.

21. Nili H, Wingfield C, Walther A, Su L, Marslen-Wilson W, Kriegeskorte N. A toolbox for representational similarity analysis. PLoS Comput Biol. 2014;10(4):e1003553. 
22. Kwok SC, Macaluso E. Scale invariance of temporal order discrimination using complex, naturalistic events. Cognition. 2015;140:111-21.

23. Gallistel CR, Gibbon J. Time, rate, and conditioning. Psychol Rev. 2000;107(2):289-344.

24. Greene MR, Baldassano C, Esteva A, Beck DM, Fei-Fei L. Visual scenes are categorized by function. J Exp Psychol Gen. 2016;145(1):82-94.

25. Aminoff EM, Toneva M, Shrivastava A, Chen X, Misra I, Gupta A, et al. Applying artificial vision models to human scene understanding. Front Comput Neurosci. 2015;9:8.

26. Lowe DG. Distinctive image features from scale-invariant keypoints. International Journal of Computer Vision. 2004;60(2):91-110.

27. Bay H, Ess A, Tuytelaars T, Van Gool L. Speeded-Up Robust Features (SURF). Computer Vision and Image Understanding. 2008;110(3):346-59.

28. Dalal N, Triggs B, editors. Histograms of oriented gradients for human detection. Computer Vision and Pattern Recognition, 2005 CVPR 2005 IEEE Computer Society Conference on; 2005: IEEE.

29. Deuker L, Bellmund JL, Navarro Schroder T, Doeller CF. An event map of memory space in the hippocampus. Elife. 2016;5.

30. Nelson SM, McDermott KB, Petersen SE. In favor of a 'fractionation' view of ventral parietal cortex: comment on Cabeza et al. Trends Cogn Sci. 2012;16(8):399-400; author reply -1.

31. Nilakantan AS, Bridge DJ, Gagnon EP, VanHaerents SA, Voss JL. Stimulation of the Posterior Cortical-Hippocampal Network Enhances Precision of Memory Recollection. Curr Biol. 2017;27(3):465-70.

32. Wang JX, Rogers LM, Gross EZ, Ryals AJ, Dokucu ME, Brandstatt KL, et al. Targeted enhancement of cortical-hippocampal brain networks and associative memory. Science. 2014;345(6200):1054-7.

33. Eichenbaum H. Time cells in the hippocampus: a new dimension for mapping memories. Nat Rev Neurosci. 2014;15(11):732-44.

34. Brodt S, Pohlchen D, Flanagin VL, Glasauer S, Gais S, Schonauer M. Rapid and independent memory formation in the parietal cortex. Proc Natl Acad Sci U S A. 2016;113(46):13251-6.

35. Kyle CT, Smuda DN, Hassan AS, Ekstrom AD. Roles of human hippocampal subfields in retrieval of spatial and temporal context. Behav Brain Res. 2015;278:549-58.

36. Copara MS, Hassan AS, Kyle CT, Libby LA, Ranganath C, Ekstrom AD. Complementary roles of human hippocampal subregions during retrieval of spatiotemporal context. J Neurosci. 2014;34(20):6834-42.

37. Lositsky O, Chen J, Toker D, Honey CJ, Shvartsman M, Poppenk JL, et al. Neural pattern change during encoding of a narrative predicts retrospective duration estimates. Elife. 2016;5.

38. Rotem-Turchinski N, Ramaty A, Mendelsohn A. The opportunity to choose enhances long-term episodic memory. Memory. 2018:1-10.

39. Chen HY, Gilmore AW, Nelson SM, McDermott KB. Are There Multiple Kinds of Episodic Memory? An fMRI Investigation Comparing Autobiographical and Recognition Memory Tasks. J Neurosci. 2017;37(10):2764-75.

40. Wang F, Diana RA. Temporal context in human fMRI. Current Opinion in Behavioral Sciences. 2017;17:57-64.

41. Moscovitch M, Cabeza R, Winocur G, Nadel L. Episodic Memory and Beyond: The Hippocampus and Neocortex in Transformation. Annu Rev Psychol. 2016;67:105-34.

42. Vincent JL, Snyder AZ, Fox MD, Shannon BJ, Andrews JR, Raichle ME, et al. Coherent 
spontaneous activity identifies a hippocampal-parietal memory network. J Neurophysiol. 2006;96(6):3517-31.

919 43. Kwok SC, Macaluso E. Immediate memory for "when, where and what": Short-delay retrieval using dynamic naturalistic material. Hum Brain Mapp. 2015;36(7):2495-513.

44. Long NM, Kahana MJ. Hippocampal contributions to serial-order memory. Hippocampus. 2018;0(ja).

45. Rossi S, Hallett M, Rossini PM, Pascual-Leone A, Safety of TMSCG. Safety, ethical considerations, and application guidelines for the use of transcranial magnetic stimulation in clinical practice and research. Clinical neurophysiology : official journal of the International Federation of Clinical Neurophysiology. 2009;120(12):2008-39.

927 46. Bonni S, Veniero D, Mastropasqua C, Ponzo V, Caltagirone C, Bozzali M, et al. TMS evidence for 928 a selective role of the precuneus in source memory retrieval. Behav Brain Res. 2015;282:70-5.

929 47. Kraft A, Dyrholm M, Kehrer S, Kaufmann C, Bruening J, Kathmann N, et al. TMS over the right 930 precuneus reduces the bilateral field advantage in visual short term memory capacity. Brain Stimul. 931 2015;8(2):216-23.

932 48. Mancini M, Mastropasqua C, Bonni S, Ponzo V, Cercignani M, Conforto S, et al. Theta Burst 933 Stimulation of the Precuneus Modulates Resting State Connectivity in the Left Temporal Pole. Brain 934 Topogr. 2017;30(3):312-9.

935 49. Friston KJ, Penny W, Phillips C, Kiebel S, Hinton G, Ashburner J. Classical and Bayesian inference 936 in neuroimaging: theory. Neuroimage. 2002;16(2):465-83.

937 50. Penny W, Holmes A. Random-Effects Analysis. In: Frackowiak SJR, Friston JK, Frith DC, Dolan

938 JR, Price JC, Zeki S, et al., editors. Human Brain Function. Random-Effects Analysis. Second Edition 939 ed: Elsevier; 2004. p. pp.843-50.

940 51. Talairach J, Tournoux P. Co-planar stereotaxic atlas of the human brain. 3-Dimensional proportional 941 system: an approach to cerebral imaging: Thieme; 1988.

942 52. Esterman M, Tamber-Rosenau BJ, Chiu YC, Yantis S. Avoiding non-independence in fMRI data 943 analysis: leave one subject out. Neuroimage. 2010;50(2):572-6.

944 53. Tzourio-Mazoyer N, Landeau B, Papathanassiou D, Crivello F, Etard O, Delcroix N, et al. 945 Automated anatomical labeling of activations in SPM using a macroscopic anatomical parcellation of 946 the MNI MRI single-subject brain. Neuroimage. 2002;15(1):273-89.

947 54. Viola P, Jones MJ. Robust real-time face detection. International Journal of Computer Vision. $948 \quad$ 2004;57(2):137-54. 